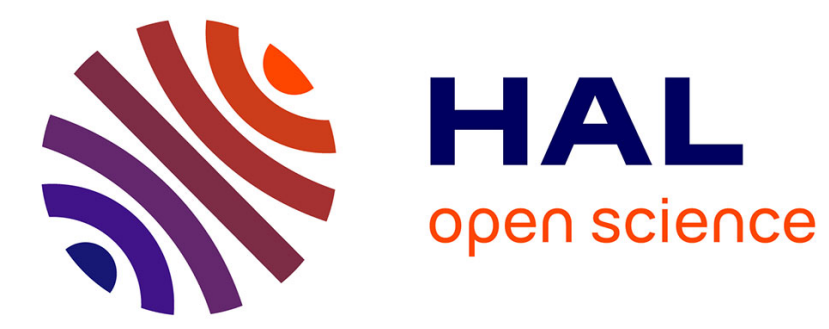

\title{
A formal series approach to the center manifold theorem
}

\author{
François Castella, Philippe Chartier, Julie Sauzeau
}

\section{To cite this version:}

François Castella, Philippe Chartier, Julie Sauzeau. A formal series approach to the center manifold theorem. Foundations of Computational Mathematics, 2018, 18 (6), pp.1397-1434. 10.1007/s10208017-9371-y . hal-01279715v2

\section{HAL Id: hal-01279715 \\ https://hal.inria.fr/hal-01279715v2}

Submitted on 15 Apr 2016

HAL is a multi-disciplinary open access archive for the deposit and dissemination of scientific research documents, whether they are published or not. The documents may come from teaching and research institutions in France or abroad, or from public or private research centers.
L'archive ouverte pluridisciplinaire HAL, est destinée au dépôt et à la diffusion de documents scientifiques de niveau recherche, publiés ou non, émanant des établissements d'enseignement et de recherche français ou étrangers, des laboratoires publics ou privés. 


\title{
A formal series approach to the center manifold theorem
}

\author{
F. Castella*, Ph. Chartier, J. Sauzeau ${ }^{\ddagger}$
}

April 15, 2016

\begin{abstract}
This paper considers near-equilibrium systems of ordinary differential equations with explicit separation of the slow and stable manifolds. Formal B-series like those previously used to analyze highly-oscillatory systems or to construct modified equations are employed here to construct expansions of the change of variables, the center invariant manifold and the reduced model. The new approach may be seen as a process of reduction to a normal form, with the main advantage, as compared to the standard view conveyed by the celebrated center manifold theorem, that it is possible to recover the complete solution at any time through an explicit change of variables.
\end{abstract}

Keywords Center manifold, stable manifold, slow manifold, shadowing principle, change of variables, B-series, trees, composition product, normal form, model reduction.

Mathematics Subject Classification (2000) 34C29, 65L06, 34D20, 70H05, 79K65.

\section{Introduction}

In the neighborhood of an equilibrium point of a dynamical system, the center manifold is made of orbits which are neither attracted by the stable manifold nor repulsed by the unstable one. A preliminary step when studying equilibria is usually to linearize the system: the phase-space can then be decomposed as the direct sum of the stable and unstable eigenspaces of the linear operator. The former corresponds to eigenvectors associated with eigenvalues having negative real parts whereas the latter is formed by eigenvectors associated with eigenvalues having positive real parts. If the equilibrium under consideration is hyperbolic (i.e. if all eigenvalues have nonzero real parts), the behavior of the dynamical system is fully characterized. If not however, i.e. if some eigenvalues have null real parts, then the corresponding eigenvectors give rise to a center manifold. If these eigenvalues not only have null real parts but are zero, then the center manifold is called a slow manifold. This is the situation we consider in this paper and which appears, for instance, in a number of applications to population dynamics ${ }^{1}$ [AR94, CHL09]. More precisely, we are concerned with partitioned systems of differential equations of the form

$$
\left\{\begin{array}{l}
\dot{x}=\varepsilon f(x, z) \\
\dot{z}=-\Lambda z+\varepsilon g(x, z)
\end{array}\right.
$$

\footnotetext{
*IRMAR, University of Rennes 1, Rennes, France. Email: francois.castella@univ-rennes1.fr

${ }^{\dagger}$ INRIA Rennes, ENS Rennes, Campus Ker-Lann, av. Robert Schumann, 35170 Bruz, France. Email: Philippe.Chartier@inria.fr

${ }^{\ddagger}$ IRMAR, University of Rennes 1, Rennes, France. Email: julie.sauzeau@univ-rennes1.fr

${ }^{1} \mathrm{~A}$ number of recent publications consider the more involved situation where the matrix $\Lambda$ exhibits additionally a periodic dependency in time [PA96, CCS].
} 
with initial condition $(x(0), z(0))=\left(x_{0}, z_{0}\right) \in \mathbb{R}^{n} \times \mathbb{R}^{m}$ and where $\Lambda \in \mathbb{R}^{m \times m}$ is a diagonal matrix with strictly positive diagonal elements $\lambda_{i}, i=1, \ldots, m$. In essence, existing theorems from the literature address the possibility that the $\lambda_{i}$ are different. In this first paper however, we analyze the technically less demanding situation where all $\lambda_{i}$ coincide. Besides and without additional loss of generality, we fix to 1 their common value.

\subsection{A statement of the center manifold theorem}

The celebrated center-manifold theorem -see for instance [Car81]- assumes here the following wording:

Theorem 1.1 (Center manifold theorem and shadowing principle) Let $B_{R} \subset \mathbb{R}^{n} \times \mathbb{R}^{m}$ be the ball of radius $R$ centered at the origin. For all $R>0$, there exists $\varepsilon^{*}>0$ and $T>0$ such that the solution $(x(t), z(t))$ of (1.1) exists for all $0<\varepsilon<\varepsilon^{*}$, all $0 \leq t \leq T / \varepsilon$ and all initial condition $\left(x_{0}, z_{0}\right) \in B_{R}$. Moreover, there exists a ( $\varepsilon$-dependent) function $h: \mathbb{R}^{n} \rightarrow \mathbb{R}^{m}$, defined for all $0<\varepsilon<\varepsilon^{*}$, such that

$$
\mathcal{M}=\left\{(x, z) \in \mathbb{R}^{n} \times \mathbb{R}^{m} ; z=\varepsilon h(x)\right\}
$$

is an invariant manifold of (1.1) in the following sense: if $\left(x_{0}, z_{0}\right) \in \mathcal{M} \cap B_{R}$, then $(x(t), z(t)) \in \mathcal{M}$ for all $t \in[0, T / \varepsilon]$. Denoting $\varphi_{t}$ the exact flow of the reduced equation

$$
\dot{u}=\varepsilon f(u, \varepsilon h(u))
$$

one can assert that there exists $\mu>0$ such that for all $\left(x_{0}, z_{0}\right) \in B_{R}$

$$
\forall t \in\left[0, \frac{T}{\varepsilon}\right], z(t)=\varepsilon h\left(\varphi_{t}\left(x_{0}\right)\right)+\mathcal{O}\left(e^{-\mu t}\right) .
$$

Furthermore, there exists a modified initial data $x_{0}^{\varepsilon} \in \mathbb{R}^{n}$ such that

$$
\forall t \in\left[0, \frac{T}{\varepsilon}\right], x(t)=\varphi_{t}\left(x_{0}^{\varepsilon}\right)+\mathcal{O}\left(e^{-\mu t}\right) \quad \text { and } \quad z(t)=\varepsilon h\left(\varphi_{t}\left(x_{0}^{\varepsilon}\right)\right)+\mathcal{O}\left(e^{-\mu t}\right) .
$$

Finally, if $T=+\infty$ for some $R$, then equations (1.3) are satisfied for all $t \geq 0$.

The interest of the center manifold theorem is apparent: provided the function $h$ and the value of $x_{0}^{\varepsilon}$ are known, the dynamics of (1.1) is asymptotically described by the reduced system

$$
\left\{\begin{aligned}
\dot{x}^{\infty} & =\varepsilon f\left(x^{\infty}, \varepsilon h\left(x^{\infty}\right)\right) \\
z^{\infty} & =\varepsilon h\left(x^{\infty}\right)
\end{aligned}\right.
$$

with modified initial condition $x^{\infty}(0)=x_{0}^{\varepsilon}$. Besides, it is not hard in our setting to obtain a formal $\varepsilon$-expansion of $h$ from the partial differential equation

$$
\varepsilon \partial_{x} h(x) f(x, \varepsilon h(x))=-h(x)+\varepsilon g(x, \varepsilon h(x)) .
$$




\subsection{Scope of the paper}

Theorem 1.1 states the existence of a function $h$, and a perturbed initial condition $x_{0}^{\varepsilon}$, allowing for a reformulation of the dynamics of (1.1) with an asymptotically exponentially small discrepancy. Nevertheless, whereas $h$ may be approached iteratively through (1.4), nothing is said on how to construct $x_{0}^{\varepsilon}$. Obtaining an expression of $x_{0}^{\varepsilon}$ in terms of $x_{0}$ and $z_{0}$ is hence clearly part of our motivation, as it appears as a prerequisite for obtaining an exponentially-close $x$-approximation. An additional strong motivation for this work stems from the need for a better approximation of the transient phase, that is to say the "small" interval of time close to the initial time where the solution $(x(t), z(t))$ undergoes a rapid variation. From this point of view, Theorem 1.1 is indeed largely unsatisfactory given that $e^{-\mu t} \approx 1$ for small values of $t$. Our main contribution in this work is to show that it is possible to complement the reduced center-manifold equation in $x^{\infty}$ with a second equation in $\tilde{y}$, leading to a system

$$
\left\{\begin{array}{l}
\dot{x}^{\infty}=\varepsilon f\left(x^{\infty}, \varepsilon h\left(x^{\infty}\right)\right) \\
\dot{\tilde{y}}=\varepsilon G\left(x^{\infty}, \tilde{y}\right)
\end{array},\right.
$$

whose solution for the initial condition $\left(x^{\infty}(0), \tilde{y}(0)\right)=\left(x_{0}^{\varepsilon}, y_{0}\right)$ can then be used to compute the solution $(x(t), z(t))$ of (1.1) exactly for all time. All the transformations required to prove this result are obtained via B-series, introduced as such in [HLW06] and pioneered by J.C. Butcher [But72, But87]. B-series are series expansions in powers of $\varepsilon$, which allow for the effective explicit computation of, for instance, the exact solution of (1.1). They involve two types of terms: on the one hand, scalar coefficients $^{2}$ which are universal (in the sense that they are independent of the specific functions $f$ and $g$ ) and encode the intrinsic properties of the class of systems being studied, and on the other hand, socalled elementary differentials (composed of various derivatives of $f$ and $g$ and constructed in a very simple way). This type of representation has proved to be very helpful to construct modified equations [CHV10] or to analyze highly-oscillatory differential equations [CMSS10, CMSS12b]. The B-series approach may also be applied to the derivation of estimates for the remainder (the terms $\mathcal{O}\left(e^{-\mu t}\right)$ of Theorem 1.1, see for instance [CMSS15, CMSS12a]). This aspect will be the subject of a forthcoming paper and will not be to addressed any longer here. It is worth mentioning that word-series, though less general than B-series, constitute an appealing alternative to B-series, as they are much simpler to compose. In a recent series of papers [MSS16a, MSS16b, MSS16c], A. Murua and J.M. Sanz-Serna resort to word-series to compute normal forms of a large class of systems including (1.1). Their works share many similarities with the present one: in particular [MSS16a] considers the same transport equation as in Section 2.4 of this paper, or Section 2.4 of [CMSS12b]. However, it differs in that the adjoined initial conditions are not prescribed at the same time. This choice manifests itself in the resulting normal equations (and will be discussed in greater details in Remark 2.26): the form obtained here in Theorem 2.25 and sketched above is close to the standard one of Theorem 1.1 and retains its main advantage, i.e. the reduction of dimensionality: starting from a problem posed in $\mathbb{R}^{n} \times \mathbb{R}^{m}$, the normal form obtained here is a partially decoupled system.

The main ideas of the paper are exposed in Section 2. At first, we shall motivate in Subsection 2.1 the use of B-series, with a direct attempt at deriving the formal expansions of the solution of (1.1). We will then present, in Subsection 2.2, the trees and elementary differentials required in this context and introduce Taylor-indexed B-series in Subsection 2.3, together with some of their features which are essential to the subsequent analysis. Subsection 2.4 is devoted to the main properties of the B-series

\footnotetext{
${ }^{2}$ The coefficients we consider here are time-dependent.
} 
coefficients of the exact solution of (1.1). In particular, it is shown therein that they are amenable to a time-scale separation after which they obey a transport equation. The transformation that maps $x_{0}$ to $x_{0}^{\varepsilon}$ is also explicitly defined in this part. Equations of the center-manifold dynamics are then derived in Subsection 2.5, where a theorem similar to Theorem 1.1 is given. We conclude this second section with the statement of the main result of the paper in Subsection 2.6.

In Section 3, we illustrate numerically the main outcomes of our analysis on two simple examples. For both systems, we derive a third-order approximation of all transformations considered and show that the results of our theorems are indeed valid up to errors of fourth-order with respect to (w.r.t.) $\varepsilon$.

\section{Center manifold via B-series}

In [CMSS10] and [CMSS12b], where highly-oscillatory systems are studied, the analysis leans on a Fourier expansion of the periodic vector field. Here, the corresponding ad-hoc tool is a Taylor expansion in the neighbourhood of $z=0$. We hereafter explain how to proceed.

Through the change of variable $z(t)=e^{-t \Lambda} y(t)$, system (1.1) can be equivalently rewritten as

$$
\left\{\begin{array}{ll}
\dot{x}=\varepsilon f\left(x, e^{-t \Lambda} y\right) & :=\varepsilon F_{t \Lambda}(x, y) \\
\dot{y}=\varepsilon e^{t \Lambda} g\left(x, e^{-t \Lambda} y\right) & :=\varepsilon G_{t \Lambda}(x, y)
\end{array},\right.
$$

with initial condition $(x(0), y(0))=\left(x_{0}, z_{0}\right)$. Assuming that both $f$ and $g$ are real-analytic w.r.t. the $y$-variable, we have the Taylor expansions

$$
\begin{aligned}
& f(x, z)=f(x, 0)+\sum_{\mathbf{k} \in \mathbb{N}^{m}} \frac{1}{\mathbf{k} !} D_{z}^{\mathbf{k}} f(x, 0) z^{\mathbf{k}}, \\
& g(x, z)=g(x, 0)+\sum_{\mathbf{k} \in \mathbb{N}^{m}} \frac{1}{\mathbf{k} !} D_{z}^{\mathbf{k}} g(x, 0) z^{\mathbf{k}},
\end{aligned}
$$

where $\mathbf{k} !=k_{1} ! \cdots k_{n}$ ! and where

$$
\left[D_{z}^{\mathbf{k}} f(x, 0) z^{\mathbf{k}}\right]_{i}=\frac{\partial^{\mid \mathbf{k}} f_{i}(x, 0)}{\partial z_{1}^{k_{1}} \ldots \partial z_{n}^{k_{n}}} z_{1}^{k_{1}} \ldots z_{n}^{k_{n}} \quad \text { with } \quad|\mathbf{k}|=k_{1}+\ldots+k_{n}
$$

and similarly for $g$. In particular, one has

$$
D_{z}^{\mathbf{k}} f(x, 0)\left(e^{-t \Lambda} y\right)^{\mathbf{k}}=e^{-t(\mathbf{k} \cdot \lambda)} D_{z}^{\mathbf{k}} f(x, 0) y^{\mathbf{k}} \quad \text { with } \quad \mathbf{k} \cdot \lambda=\sum_{i=1}^{n} k_{i} \lambda_{i}
$$

so that one can eventually write

$$
\begin{aligned}
& F_{t \Lambda}(x, y)=f(x, 0)+\sum_{\mathbf{k} \in \mathbb{N}^{m}} \frac{e^{-t(\mathbf{k} \cdot \lambda)}}{\mathbf{k} !} D_{z}^{\mathbf{k}} f(x, 0) y^{\mathbf{k}} \\
& G_{t \Lambda}(x, y)=e^{t \Lambda} g(x, 0)+\sum_{\mathbf{k} \in \mathbb{N}^{m}} \frac{e^{-t(\mathbf{k} \cdot \lambda)}}{\mathbf{k} !} e^{t \Lambda} D_{z}^{\mathbf{k}} g(x, 0) y^{\mathbf{k}}
\end{aligned}
$$


expressions which can be further simplified by taking into account that all $\lambda_{i}$ are assumed to be equal to 1

$$
\begin{aligned}
& F_{t}(x, y)=f(x, 0)+\sum_{\mathbf{k} \in \mathbb{N}^{m}} \frac{e^{-t|\mathbf{k}|}}{\mathbf{k} !} D_{z}^{\mathbf{k}} f(x, 0) y^{\mathbf{k}} \\
& G_{t}(x, y)=e^{t} g(x, 0)+\sum_{\mathbf{k} \in \mathbb{N}^{m}} \frac{e^{-t(|\mathbf{k}|-1)}}{\mathbf{k} !} D_{z}^{\mathbf{k}} g(x, 0) y^{\mathbf{k}} .
\end{aligned}
$$

To sum up, the equations analyzed throughout this paper are of the following form

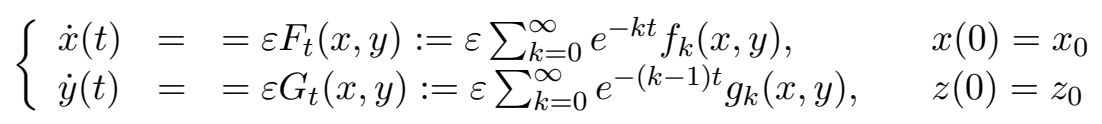

where we have denoted ${ }^{3}$

$$
f_{k}(x, z)=\sum_{\mathbf{k} \in \mathbb{N}^{m},|\mathbf{k}|=k} \frac{1}{\mathbf{k} !}\left(D_{z}^{\mathbf{k}} f\right)(x, 0) z^{\mathbf{k}} \quad \text { and } \quad g_{k}(x, z)=\sum_{\mathbf{k} \in \mathbb{N}^{m},|\mathbf{k}|=k} \frac{1}{\mathbf{k} !}\left(D_{z}^{\mathbf{k}} g\right)(x, 0) z^{\mathbf{k}} .
$$

Since we shall not study the convergence of the series manipulated in this paper $^{4}$, we will furthermore assume that the series in (2.4) are finite, i.e. that $f$ and $g$ are polynomials.

\subsection{Expansion of the transient solution}

In order to motivate the introduction of trees and B-series in next subsection, we first derive a few terms of the formal $\varepsilon$-expansion of the components $(x(t), y(t))$ of the solution and this is done by considering equations (2.4) in their integral form:

$$
\begin{aligned}
& x(t)=x_{0}+\varepsilon \int_{0}^{t} \sum_{k \geq 0} e^{-k s} f_{k}(x(s), y(s)) d s=x_{0}+\mathcal{O}(\varepsilon), \\
& y(t)=y_{0}+\varepsilon \int_{0}^{t} \sum_{k \geq 0} e^{-(k-1) s} g_{k}(x(s), y(s)) d s=y_{0}+\mathcal{O}(\varepsilon) .
\end{aligned}
$$

Introducing these expressions in the right-hand side of the equations, we now obtain

$$
\begin{aligned}
& x(t)=x_{0}+\varepsilon \sum_{k \geq 0}\left(\int_{0}^{t} e^{-k s} d s\right) f_{k}\left(x_{0}, y_{0}\right)+\mathcal{O}\left(\varepsilon^{2}\right), \\
& y(t)=y_{0}+\varepsilon \sum_{k \geq 0}\left(\int_{0}^{t} e^{-(k-1) s} d s\right) g_{k}\left(x_{0}, y_{0}\right)+\mathcal{O}\left(\varepsilon^{2}\right) .
\end{aligned}
$$

\footnotetext{
${ }^{3}$ Note that for all $\lambda \in \mathbb{R}, f_{k}(x, \lambda z)=\lambda^{k} f_{k}(x, z)$ and similarly for $g_{k}$.

${ }^{4}$ This will be done in a forthcoming paper.
} 
Omitting the argument $\left(x_{0}, y_{0}\right)$ of the various functions, a third iteration then leads to

$$
\begin{aligned}
x(t)= & x_{0}+\varepsilon \sum_{k \geq 0}\left(\int_{0}^{t} e^{-k s} d s\right) f_{k}+\varepsilon^{2} \sum_{k, r}\left(\int_{0}^{t} e^{-k s} \int_{0}^{s} e^{-r \sigma} d \sigma d s\right)\left(\partial_{x} f_{k}\right) f_{r} \\
& +\varepsilon^{2} \sum_{k, r}\left(\int_{0}^{t} e^{-k s} \int_{0}^{s} e^{-(r-1) \sigma} d \sigma d s\right)\left(\partial_{y} f_{k}\right) g_{r}+\mathcal{O}\left(\varepsilon^{3}\right) \\
y(t)= & y_{0}+\varepsilon \sum_{k \geq 0}\left(\int_{0}^{t} e^{-(k-1) s} d s\right) g_{k}+\varepsilon^{2} \sum_{k, r}\left(\int_{0}^{t} e^{-(k-1) s} \int_{0}^{s} e^{-r \sigma} d \sigma d s\right)\left(\partial_{x} g_{k}\right) f_{r} \\
& +\varepsilon^{2} \sum_{k, r}\left(\int_{0}^{t} e^{-(k-1) s} \int_{0}^{s} e^{-(r-1) \sigma} d \sigma d s\right)\left(\partial_{y} g_{k}\right) g_{r}+\mathcal{O}\left(\varepsilon^{3}\right) .
\end{aligned}
$$

It is clear that this procedure à la Picard can be iterated to obtain the $\varepsilon^{3}, \varepsilon^{4}, \ldots$ terms of the expansions of $x(t)$ and $y(t)$. However, the growing complexity of the expressions arising in the process impedes a systematic construction. This is the reason why we shall use Taylor-indexed rooted trees and associated elementary differentials as a mean to derive the sought-after series with explicit inductions.

\subsection{Taylor-indexed bicoloured trees and elementary differentials}

We consider bi-coloured rooted trees where black vertices refer to function $f$, white vertices to function $g$ and where each vertex has been labelled with a index $k \geq 0$ associated to the $k$-th terms $f_{k}$ and $g_{k}$ in the Taylor expansions of $f$ and $g$. For the sake of simplicity, we use hereafter the word tree.

Definition 2.1 The set of Taylor-index bicoloured trees (or simply trees) $\mathcal{T}=\mathcal{T}_{\bullet} \cup \mathcal{T}_{\circ}$ is defined recursively as follows:

1. For any index $k \in \mathbb{N}$, the tree with a single indexed vertex $\bullet_{k}$ belongs to $\mathcal{T}_{\bullet}$ and the tree with a single indexed vertex $\circ_{k}$ belongs to $\mathcal{T}_{\circ}$.

2. For any index $k \in \mathbb{N}$, any $(p, q) \in \mathbb{N} \times \mathbb{N}$ with $q \leq k$, any $\left(u_{1}, \ldots, u_{p}\right) \in \mathcal{T}_{\bullet}^{p}$ and $\left(v_{1}, \ldots, v_{q}\right) \in$ $\mathcal{T}_{0}^{q}$, the tree $\left[u_{1}, \ldots, u_{p}, v_{1}, \ldots, v_{q}\right]_{\bullet_{k}}$ obtained by connecting the roots of $u_{1}, \ldots, u_{p}, v_{1}, \ldots, v_{q}$ to a new root $\bullet_{k}$, belongs to $\mathcal{T}_{\text {. }}$. Similarly, $\left[u_{1}, \ldots, u_{p}, v_{1}, \ldots, v_{q}\right]_{\circ_{k}} \in \mathcal{T}_{\text {o. }}$.

For homogeneity, the empty trees $\emptyset_{x}$ and $\emptyset_{y}$ will sometimes be used to denote $\mathcal{F}_{\emptyset_{x}}(x, y)=x$ and $\mathcal{F}_{\emptyset_{y}}(x, y)=y$, and we shall write accordingly $\overline{\mathcal{T}}_{\bullet}=\mathcal{T}_{\bullet} \cup\left\{\emptyset_{x}\right\}, \overline{\mathcal{T}}_{\circ}=\mathcal{T}_{\circ} \cup\left\{\emptyset_{y}\right\}$ and $\overline{\mathcal{T}}=\overline{\mathcal{T}}_{\bullet} \cup \overline{\mathcal{T}}_{\circ}$.

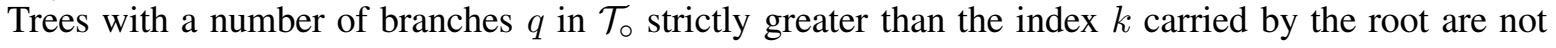
permitted. This comes from the fact that the $q$-th derivatives of $f_{k}$ and $g_{k}$ with respect to $y$ vanish identically for $q>k$, given that both $f_{k}$ and $g_{k}$ are $k$-linear maps with respect to $y$. For instance, $\rho_{0}^{1} \in \mathcal{T}_{\bullet}$ but $\rho_{0}^{1} \notin \mathcal{T}$. This will become completely clear with Definition 2.3.

The order of a tree $w \in \mathcal{T}$, denoted $|w|$, is its number of vertices. The symmetry factor measures how symmetric the tree looks like and is defined as follows: note that vertices with different labels are distinguished.

Definition 2.2 The symmetry factor is defined recursively on $\mathcal{T}$ as follows:

1. For all $k \in \mathbb{N}, \sigma_{\bullet_{k}}=\sigma_{\circ_{k}}=1$. 
2. Let $w$ be of the form either $\left[u_{1}^{\mu_{1}}, \ldots, u_{p}^{\mu_{p}}, v_{1}^{\nu_{1}}, \ldots, v_{q}^{\nu_{q}}\right]_{\bullet_{k}}$ or $\left[u_{1}^{\mu_{1}}, \ldots, u_{p}^{\mu_{p}}, v_{1}^{\nu_{1}}, \ldots, v_{q}^{\nu_{q}}\right]_{\mathrm{o}_{k}}$, where trees $u_{i}$ and $v_{j}$ are assumed to be pairwise distinct, and where exponents $\mu_{i}$ and $\nu_{j}$ indicate that $u_{i}$ and $v_{j}$ are repeated $\mu_{i}$ and $\nu_{j}$ times. Then

$$
\sigma_{w}=\prod_{i=1}^{p} \mu_{i} ! \sigma_{u_{i}}^{\mu_{i}} \prod_{j=1}^{q} \nu_{j} ! \sigma_{v_{j}}^{\nu_{j}}
$$

Finally, to each tree we associate an elementary differential, i.e. a function from $\mathbb{R}^{n} \times \mathbb{R}^{m}$ to either $\mathbb{R}^{n}$ or $\mathbb{R}^{m}$, depending on whether the root is black or white. The label of the root then determines which function is differentiated.

Definition 2.3 The elementary differentials associated to trees of $\mathcal{T}$ are defined recursively as follows:

1. For all $k \in \mathbb{N}, \mathcal{F}_{\bullet_{k}}(x, y)=f_{k}(x, y)$ and $\mathcal{F}_{\mathrm{o}_{k}}(x, y)=g_{k}(x, y)$.

2. If $u=\left[u_{1}, \ldots, u_{p}, v_{1}, \ldots, v_{q}\right]_{\bullet_{k}}$, then

$$
\mathcal{F}_{u}(x, y)=\left(\partial_{x}^{p} \partial_{y}^{q} f_{k}\right)(x, y)\left(\mathcal{F}_{u_{1}}(x, y), \ldots, \mathcal{F}_{u_{p}}(x, y), \mathcal{F}_{v_{1}}(x, y), \ldots, \mathcal{F}_{v_{q}}(x, y)\right),
$$

and if $v=\left[u_{1}, \ldots, u_{p}, v_{1}, \ldots, v_{q}\right]_{\circ_{k}}$, then

$$
\mathcal{F}_{v}(x, y)=\left(\partial_{x}^{p} \partial_{y}^{q} g_{k}\right)(x, y)\left(\mathcal{F}_{u_{1}}(x, y), \ldots, \mathcal{F}_{u_{p}}(x, y), \mathcal{F}_{v_{1}}(x, y), \ldots, \mathcal{F}_{v_{q}}(x, y)\right) .
$$

According to previous definitions, the truncated expansions (2.5) and (2.6) can be rewritten as

$$
\begin{aligned}
& x(t)=x_{0}+\sum_{u \in \mathcal{T}_{\bullet},|u| \leq 2} \varepsilon^{|u|} \frac{\alpha_{u}(t)}{\sigma_{u}} \mathcal{F}_{u}\left(x_{0}, y_{0}\right)+\mathcal{O}\left(\varepsilon^{3}\right), \\
& y(t)=y_{0}+\sum_{v \in \mathcal{T}_{o},|v| \leq 2} \varepsilon^{|v|} \frac{\alpha_{v}(t)}{\sigma_{v}} \mathcal{F}_{v}\left(x_{0}, y_{0}\right)+\mathcal{O}\left(\varepsilon^{3}\right),
\end{aligned}
$$

with

$$
\alpha_{\bullet_{k}}(t)=\int_{0}^{t} e^{-k s} d s, \alpha_{\boldsymbol{\sigma}_{\mathrm{k}}^{\mathrm{r}}}(t)=\int_{0}^{t} \int_{0}^{s} e^{-k s-r \sigma} d \sigma d s, \alpha_{\substack{\boldsymbol{o}_{\mathrm{k}}^{\mathrm{r}} \\(t)}}^{s}(t)=\int_{0}^{s} \int_{0}^{-k s-(r-1) \sigma} d \sigma d s,
$$

and

$$
\alpha_{\mathrm{o}_{k}}(t)=\int_{0}^{t} e^{-(k-1) s} d s, \alpha_{\delta_{\mathrm{k}}^{\mathrm{r}}}(t)=\int_{0}^{t} \int_{0}^{s} e^{-(k-1) s-r \sigma} d \sigma d s, \alpha_{\substack{\mathrm{ok}_{\mathrm{k}}^{\mathrm{r}} \\ \mathrm{r}}}(t)=\int_{0}^{t} \int_{0}^{s} e^{-(k-1) s-(r-1) \sigma} d \sigma d s .
$$

\subsection{Taylor-indexed partitioned B-series}

In this subsection, we now consider Taylor-indexed partitioned B-series, which will constitute the main tool employed in this paper. For brevity again, we shall simply call them B-series.

Definition 2.4 A Taylor-indexed partitionned B-series (or simply B-series) with coefficients $a: \overline{\mathcal{T}} \rightarrow$ $\mathbb{C}$, is a formal expansion of the form

$$
B(a,(x, y))=\left(a_{\emptyset_{x}} x+\sum_{u \in \mathcal{T}_{\bullet}} \frac{\varepsilon^{|u|}}{\sigma_{u}} a_{u} \mathcal{F}_{u}(x, y), a_{\emptyset_{y}} y+\sum_{v \in \mathcal{T}_{\circ}} \frac{\varepsilon^{|v|}}{\sigma_{v}} a_{v} \mathcal{F}_{v}(x, y)\right)
$$


We shall incidentally write

$$
B_{\bullet}(a,(x, y))=a_{\emptyset_{x}} x+\sum_{u \in \mathcal{T}_{\bullet}} \frac{\varepsilon^{|u|}}{\sigma_{u}} a_{u} \mathcal{F}_{u}(x, y)
$$

for the component $x$ of $B(a,(x, y))$ and accordingly $B_{\circ}(a,(x, y))$ for the component $y$. Although this is not reflected in the notations, a B-series depends on the functions $f_{k}$ and $g_{k}$, and thus on $f$ and $g$, through the elementary differentials $\mathcal{F}$. According to this definition and to equations (2.4), we can write the function $(x, y) \mapsto\left(\varepsilon F_{t}(x, y), \varepsilon G_{t}(x, y)\right)$ as a B-series

$$
\left(\varepsilon F_{t}(x, y), \varepsilon G_{t}(x, y)\right)=B(\beta(t),(x, y))
$$

with coefficients $\beta$ depending on $t$ and defined as follows:

$$
\beta_{\bullet_{k}}=e^{-k t}, \beta_{\circ_{k}}=e^{-(k-1) t}, \beta_{w}=0 \text { for all } w \in \overline{\mathcal{T}} \backslash\left\{\bullet_{k}, o_{k}, k \in \mathbb{N}\right\} .
$$

Two B-series with coefficients $a$ and $b$ such that $a_{\emptyset_{x}}=a_{\emptyset_{y}}=1$ can be composed to form a new $\mathrm{B}$-series with coefficients $c$, that is to say

$$
B(b, B(a,(x, y))=B(c,(x, y)) \quad \text { with } \quad c=a * b .
$$

More precisely, each $c_{w}$ is an explicitly known polynomial of the $a_{w^{\prime}}$ and $b_{w^{\prime \prime}}$. The star product is noncommutative and the set of near-identity mappings $a \in \mathbb{C}^{\overline{\mathcal{T}}}$ with $a_{\emptyset_{x}}=a_{\emptyset_{y}}=1$ is a non-commutative group $\mathcal{G}$, named Butcher group, with unit element $\mathbb{1}$, defined by $\mathbb{1}_{\emptyset_{x}}=\mathbb{1}_{\emptyset_{y}}=1$ and $\mathbb{1}_{w}=0$ for all $w \in \mathcal{T}$. In particular, every element $a \in \mathcal{G}$ has an inverse $a^{-1} \in \mathcal{G}$ such that $a * a^{-1}=\mathbb{1}=a^{-1} * a$. Note that more generally, the star product $a * b$ is well defined for $a \in \mathcal{G}$ and $b \in \mathbb{C}^{\overline{\mathcal{T}}}$. For the sake of illustration, we give the terms of the star-product for some trees of order less than, or equal to, 3 , where $j, k, l$ are three positive integers:

$$
\begin{aligned}
& c_{\emptyset_{x}}=b_{\emptyset_{x}} \quad c_{\emptyset_{y}}=b_{\emptyset_{y}} \\
& c_{\bullet_{j}}=b_{\emptyset_{x}} a_{\bullet_{j}}+b_{\bullet_{j}} \quad c_{\circ_{j}}=b_{\emptyset_{y}} a_{\circ_{j}}+b_{\circ_{j}}
\end{aligned}
$$

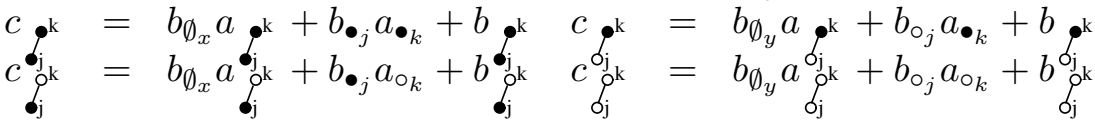

It is apparent that the color of vertices does not play a specific role, so for the trees of order 3 , we content ourselves in this brief exposition with the following mono-coloured trees:

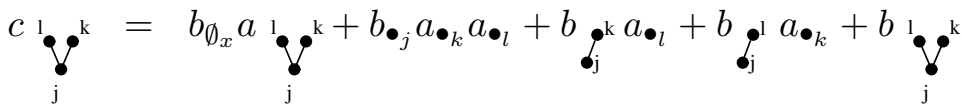

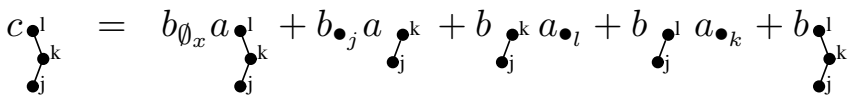

A property worth mentioning is the right-linearity of the $*$-product: if $a \in \mathcal{G}$, and $b$ and $b^{\prime}$ are in $\mathbb{C}^{\overline{\mathcal{T}}}$, then one has

$$
a *\left(b+b^{\prime}\right)=a * b+a * b^{\prime} .
$$

A further immediate property that one can easily infer from the formulae above and that we shall frequently use in the sequel is the fact that

$$
\left.(a * b)\right|_{\mathcal{T}_{\bullet}}=a *\left(\left.b\right|_{\mathcal{T}_{\bullet}}\right) \text { and }\left.(a * b)\right|_{\mathcal{T}_{\circ}}=a *\left(\left.b\right|_{\mathcal{T}_{\circ}}\right)
$$


To conclude this subsection, we now re-derive the expansion of the transient solution $(x(t), y(t))$ by using the star-product. Denoting $(x(t), y(t))=B\left(\alpha(t),\left(x_{0}, y_{0}\right)\right)$, the differential equations (2.1) can be rewritten in terms of B-series as

$$
\begin{aligned}
\frac{d}{d t} B\left(\alpha(t),\left(x_{0}, y_{0}\right)\right) & =B\left(\alpha(t) * \beta(t),\left(x_{0}, y_{0}\right)\right) \\
B\left(\alpha(0),\left(x_{0}, y_{0}\right)\right) & =B\left(\mathbb{1},\left(x_{0}, y_{0}\right)\right)
\end{aligned}
$$

Of course, this may be translated as a Cauchy problem in terms of the coefficients $\alpha \in \mathcal{G}^{\mathbb{R}}$ as

$$
\frac{d}{d t} \alpha(t)=\alpha(t) * \beta(t), \quad \alpha(0)=\mathbb{1},
$$

where $\beta$ is defined in (2.7). Note that since $\beta$ vanishes for trees of orders greater than 1 and owing to the expression of the star-product, we hereby obtain for all $k \in \mathbb{N}$

$$
\frac{d}{d t} \alpha_{\bullet_{k}}(t)=\beta_{\bullet_{k}}(t), \quad \frac{d}{d t} \alpha_{\circ_{k}}(t)=\beta_{\circ_{k}}(t),
$$

and for all $u=\left[u_{1}, \ldots, u_{p}, v_{1}, \ldots, v_{q}\right]_{\bullet_{k}} \in \mathcal{T}_{\bullet}$ and $v=\left[u_{1}, \ldots, u_{p}, v_{1}, \ldots, v_{q}\right]_{\circ_{k}} \in \mathcal{T}_{\circ}$,

$$
\frac{d}{d t} \alpha_{u}(t)=\beta_{\bullet_{k}}(t) \prod_{i=1}^{p} \alpha_{u_{i}}(t) \prod_{i=1}^{q} \alpha_{v_{i}}(t), \quad \frac{d}{d t} \alpha_{v}(t)=\beta_{\circ_{k}}(t) \prod_{i=1}^{p} \alpha_{u_{i}}(t) \prod_{i=1}^{q} \alpha_{v_{i}}(t),
$$

which, together with the initial conditions $\alpha_{w}(0)=0$, give the formulae of Subsection 2.1. Since $z_{0}=y_{0}$, we can also write

\begin{tabular}{|c|c|c|c|c|}
\hline$u$ & $\bullet_{0}$ & $\bullet_{k}$ & $\mathscr{b}_{0}^{0}$ & $\mathscr{0}_{0}^{\mathrm{r}} \quad r \geq 1$ \\
\hline$\alpha_{u}$ & $t$ & $\frac{1-e^{-k t}}{k}$ & $\frac{t^{2}}{2}$ & $\frac{-1+r t+e^{-r t}}{r^{2}}$ \\
\hline$u$ & $\mathrm{dk}^{\mathrm{r}} r \geq 1$ & $\wp_{1}^{0}$ & $\oint_{k}^{1}$ & $\delta_{\mathrm{k}}^{\mathrm{r}}(k, r) \neq(1,0), r \neq 1$ \\
\hline$\alpha_{u}$ & $\frac{r-(r+k) e^{-k t}+k e^{-(r+k) t}}{r k(r+k)}$ & $-1+t+e^{-t}$ & $\frac{1-e^{-k t}(1+k t)}{k^{2}}$ & $\frac{\frac{r-1-(k+r-1) e^{-k t}+k e^{-(k+r-1) t}}{(r-1) k(k+r-1)}}{}$ \\
\hline
\end{tabular}

$$
\begin{aligned}
& x(t)=x_{0}+\sum_{u \in \mathcal{T}_{\bullet}} \frac{\varepsilon^{|u|}}{\sigma_{u}} \alpha_{u}(t) \mathcal{F}_{u}\left(x_{0}, z_{0}\right), \\
& z(t)=e^{-t} z_{0}+\sum_{v \in \mathcal{T}_{\circ}} \frac{\varepsilon^{|v|}}{\sigma_{v}}\left(e^{-t} \alpha_{v}(t)\right) \mathcal{F}_{v}\left(x_{0}, z_{0}\right) .
\end{aligned}
$$

The first coefficients $\alpha(t)$ for trees of orders less than or equal to 2 are given in Tables 1, 2 .

Table 1: Coefficients $\alpha$ for trees $u \in \mathcal{T}_{\bullet}$ with $|u| \leq 2$ and $k \geq 1$.

\subsection{The transport equation}

This subsection contains all the technical results used to state and prove the main results of the paper. In the spirit of Lemma 2.4 and Definition 2.5 of [CMSS12b], we define polynomial functions by 


\begin{tabular}{|c|c|c|c|c|c|c|}
\hline$v$ & $\circ_{1}$ & $\circ_{k}$ & $\dot{b}_{1}^{0}$ & $\delta_{1}^{r} r \neq 0$ & $\mathrm{o}_{\mathrm{k}}^{\mathrm{r}} r \neq 0$ & $\int_{0}^{1}$ \\
\hline$\alpha_{v}$ & $t$ & $\frac{1-e^{-k^{\prime} t}}{k^{\prime}}$ & $\frac{t^{2}}{2}$ & $\frac{-1+r t+e^{-r t}}{r^{2}}$ & $\frac{r-\left(k^{\prime}+r\right) e^{-k^{\prime} t}+k^{\prime} e^{-\left(k^{\prime}+r\right) t}}{r k^{\prime}\left(k^{\prime}+r\right)}$ & $-1+e^{t}-t$ \\
\hline$v$ & $\overline{\rho_{1}^{1}}$ & $\begin{array}{l}\rho_{1}^{r} \\
\end{array}$ & $\overline{\rho_{2}^{0}}$ & $\begin{array}{l}\rho_{\mathrm{k}}^{1} \\
\quad k \geq 2\end{array}$ & ${\stackrel{\rho_{\mathrm{k}}^{\mathrm{r}}}{r}}_{r \neq 1, k \geq 2, k+r \neq 2}$ & \\
\hline$\alpha_{v}$ & $\frac{t^{2}}{2}$ & $\frac{-1+r^{\prime} t+e^{-r^{\prime} t}}{\left(r^{\prime}\right)^{2}}$ & $t-1+e^{-t}$ & $\frac{1-\left(1+k^{\prime} t\right) e^{-k^{\prime} t}}{\left(k^{\prime}\right)^{2}}$ & $\frac{r^{\prime}-\left(k^{\prime}+r^{\prime}\right) e^{-k^{\prime} t}+k^{\prime} e^{-\left(k^{\prime}+r^{\prime}\right) t}}{r^{\prime} k^{\prime}\left(k^{\prime}+r^{\prime}\right)}$ & \\
\hline
\end{tabular}

Table 2: Coefficients $\alpha$ for trees $v$ of $\mathcal{T}_{\text {。 with }}|v| \leq 2, k \neq 1, k^{\prime}=k-1$ and $r^{\prime}=r-1$.

separating the slow-time $t$ and fast-time $\tau$ variables. This requires here a little bit more care than in [CMSS12b], since we wish to keep track of the fact that coefficients $\alpha(t)$ involve exponential terms of the form $e^{-k t}$ with $k \geq-1$ only. Whereas in quasi-stroboscopic averaging as in [CMSS12b], the value at $\tau=0$ of coefficients $\gamma(t, \tau)$ was playing a major role, this is the value at $\tau=+\infty$ which here becomes central to the analysis.

Lemma 2.5 Let $w:(t, \tau) \in \mathbb{R} \times \mathbb{R} \mapsto w(t, \tau) \in \mathbb{R}$ be a continuous function which, for each fixed $\tau$, is a polynomial in $t$ and for each fixed $t$, is a polynomial in $e^{-\tau}$. If for all $t \in \mathbb{R}, w(t, t)=0$, then for all $(t, \tau) \in \mathbb{R} \times \mathbb{R}, w(t, \tau)=0$.

Definition 2.6 We say that a function $w: \mathbb{R} \times \mathbb{R} \rightarrow \mathbb{R}$ is a polynomial function if there exists a real polynomial $P \in \mathbb{R}\left[X_{1}, X_{2}\right]$ in 2 variables $X_{1}, X_{2}$, such that $w(t, \tau)=P\left(t, e^{-\tau}\right)$. Furthermore, $\eta: \mathbb{R} \times \mathbb{R} \rightarrow \mathcal{G}$ is a polynomial map, if

(i) $\eta_{w}$ is a polynomial function for each $w \in \mathcal{T}_{\bullet}$;

(ii) $e^{-\tau} \eta_{w}$ is a polynomial function for each $w \in \mathcal{T}_{\text {. }}$.

Proposition 2.7 There exists a unique polynomial map $\gamma: \mathbb{R} \times \mathbb{R} \rightarrow \mathcal{G}$ such that

$$
\alpha(t)=\gamma(t, t)
$$

where $\alpha$ is the solution of equation (2.9).

Proof: The proof proceeds by induction on the order of trees. For order 1, the assertion of the proposition can be straightforwardly checked. Now, consider $u=\left[u_{1}, \ldots, u_{p}, v_{1}, \ldots, v_{q}\right]_{\bullet_{k}} \in \mathcal{T}_{\bullet}$ and $v=\left[u_{1}, \ldots, u_{p}, v_{1}, \ldots, v_{q}\right]_{o_{k}} \in \mathcal{T}_{\circ}$, then by definition of $\alpha$ (see equations (2.9) and (2.10)), we have $\alpha_{u}(t)=\int_{0}^{t} e^{-k s} \prod_{i=1}^{p} \alpha_{u_{i}}(s) \prod_{i=1}^{q} \alpha_{v_{i}}(s) d s \quad$ and $\quad \alpha_{v}(t)=\int_{0}^{t} e^{-(k-1) s} \prod_{i=1}^{p} \alpha_{u_{i}}(s) \prod_{i=1}^{q} \alpha_{v_{i}}(s) d s$.

Using the induction hypothesis and taking into account that the set of polynomial functions is an algebra which is stable by derivation and integration (w.r.t. both $t$ and $\tau$ ), since $q \leq k$ the function

$$
e^{-k s} \prod_{i=1}^{p} \alpha_{u_{i}}(s) \prod_{i=1}^{q} \alpha_{v_{i}}(s)=e^{-(k-q)} \prod_{i=1}^{p} \alpha_{u_{i}}(s) \prod_{i=1}^{q}\left(e^{-s} \alpha_{v_{i}}(s)\right)
$$

is also of the form $\left.w(t, \tau)\right|_{\tau=t}$ for some polynomial function $w$, and so is $\alpha_{u}(t)$. A similar conclusion holds for $\alpha_{v}(t)$ if $q \leq k-1$. Now if $q=k$, we write $w(t, \tau)=P(t)+e^{-\tau} \tilde{w}(t, \tau)$ where $P(t)=$ 
$\lim _{\tau \rightarrow+\infty} w(t, \tau)$ is polynomial in $t$ and $\tilde{w}$ another polynomial function. Denoting $Q$ the unique polynomial such that $Q^{\prime}+Q=P$, we have

$$
\alpha_{v}(t)=\int_{0}^{t}\left(e^{s} P(s)+\tilde{w}(s, s)\right) d s=e^{t} Q(t)+\int_{0}^{t} \tilde{w}(s, s) d s
$$

so that $e^{-t} \alpha_{v}(t)$ is again a polynomial function.

For instance, according to Table 1 and Table 2, we have

$$
\alpha_{\boldsymbol{d}_{\mathrm{k}}^{0}}(t)=\frac{1-e^{-k t}}{k^{2}}-\frac{t e^{-k t}}{k} \text { and } \alpha_{\rho_{1}^{0}}(t)=-t+e^{t}-1,
$$

so that by substituting $t$ by $\tau$ in exponential terms, we obtain

$$
\gamma_{\mathfrak{d}_{\mathrm{k}}^{0}}(t, \tau)=\frac{1-e^{-k \tau}}{k^{2}}-\frac{t e^{-k \tau}}{k} \text { and } \gamma_{\rho_{1}^{0}}(t)=-1-t+e^{\tau} .
$$

\begin{tabular}{|c|c|c|c|c|}
\hline$u$ & $\bullet_{0}$ & $\bullet_{k}$ & $\mathscr{Q}_{0}^{0}$ & $\dot{\varphi}^{\mathrm{r}} r \geq 1$ \\
\hline$\gamma_{u}$ & $t$ & $\frac{1-e^{-k \tau}}{k}$ & $\frac{t^{2}}{2}$ & $\frac{-1+r t+e^{-r \tau}}{r^{2}}$ \\
\hline$u$ & $\mathrm{dk}^{\mathrm{e}^{\mathrm{r}}} r \geq 1$ & $\ell_{1}^{0}$ & $\rho_{\mathrm{k}}^{1}$ & 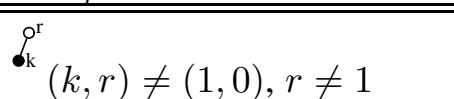 \\
\hline$\gamma_{u}$ & $\frac{r-(r+k) e^{-k \tau}+k e^{-(r+k) \tau}}{r k(r+k)}$ & $-1+t+e^{-\tau}$ & $\frac{1-e^{-k \tau}(1+k t)}{k^{2}}$ & $\frac{r-1-(k+r-1) e^{-k \tau}+k e^{-(k+r-1) \tau}}{(r-1) k(k+r-1)}$ \\
\hline
\end{tabular}

The values of $\gamma$ for trees or orders less than or equal to 2 are given in tables 3 and 4 .

\begin{tabular}{|c|c|c|c|c|c|c|}
\hline$v$ & $\circ_{1}$ & $o_{k}$ & $\dot{o}_{1}^{0}$ & $\boldsymbol{\sigma}_{1}^{r} r \neq 0$ & $\dot{\mathrm{o}}_{\mathrm{k}}^{\mathrm{r}} r \neq 0$ & $d_{0}^{1}$ \\
\hline$\gamma_{v}$ & $t$ & $\frac{1-e^{-k^{\prime} \tau}}{k^{\prime}}$ & $\frac{t^{2}}{2}$ & $\frac{-1+r t+e^{-r \tau}}{r^{2}}$ & $\frac{r-\left(k^{\prime}+r\right) e^{-k^{\prime} \tau}+k^{\prime} e^{-\left(k^{\prime}+r\right) \tau}}{r k^{\prime}\left(k^{\prime}+r\right)}$ & $-1+e^{\tau}-t$ \\
\hline$v$ & $\rho_{1}^{1}$ & $\delta_{1}^{r} \quad r \neq 1$ & $\delta_{2}^{0}$ & $\delta_{\mathrm{k}}^{1} \quad k \geq 2$ & $\delta^{\rho_{\mathrm{k}}^{\mathrm{r}}} r \neq 1, k \geq 2, k+r \neq 2$ & \\
\hline$\gamma_{v}$ & $\frac{t^{2}}{2}$ & $\frac{-1+r^{\prime} t+e^{-r^{\prime} \tau}}{\left(r^{\prime}\right)^{2}}$ & $t-1+e^{-\tau}$ & $\frac{1-\left(1+k^{\prime} t\right) e^{-k^{\prime} \tau}}{\left(k^{\prime}\right)^{2}}$ & $\frac{r^{\prime}-\left(k^{\prime}+r^{\prime}\right) e^{-k^{\prime} \tau}+k^{\prime} e^{-\left(k^{\prime}+r^{\prime}\right) \tau}}{r^{\prime} k^{\prime}\left(k^{\prime}+r^{\prime}\right)}$ & \\
\hline
\end{tabular}

Table 3: Coefficients $\gamma$ for trees $u \in \mathcal{T}_{\bullet}$ with $|u| \leq 2$ and $k \geq 1$.

Table 4: Coefficients $\gamma$ for trees $v$ of $\mathcal{T}_{\circ}$ with $|v| \leq 2, k \neq 1, k^{\prime}=k-1$ and $r^{\prime}=r-1$.

Proposition 2.8 If $\gamma \in \mathcal{G}^{\mathbb{R} \times \mathbb{R}}$ is defined as in (2.13), then $\gamma(0,0)=\mathbb{1}$ and

$$
\forall(t, \tau) \in \mathbb{R} \times \mathbb{R}, \quad \partial_{t} \gamma(t, \tau)+\partial_{\tau} \gamma(t, \tau)=\gamma(t, \tau) * \beta(\tau) .
$$

Proof: By virtue of the chain rule and equation (2.9), equation (2.14) is satisfied for $\tau=t$. The mapping $\gamma$ being polynomial, Lemma (2.5) allows to assert that equation (2.14) is actually satisfied for all $(t, \tau)$. Finally, $\gamma(0,0)=\alpha(0)=\mathbb{1}$.

In contrast with the general situation where equation (2.14) may have infinitely many solutions with the mere initial condition $\gamma(0,0)=\mathbb{1}$, the polynomial nature of $\gamma$ ensures here that there is only 
one. Actually, uniqueness can be ensured by prescribing the value of $\gamma$ at $(0,0)$, as in [CMSS12b], or at $\left(0, \tau_{0}\right)$, as in [MSS16a]. Since the asymptotic dynamics of $B_{\bullet}\left(\gamma(t, \tau),\left(x_{0}, y_{0}\right)\right)$ is here attained for $\tau=+\infty$, we have to address the question of uniqueness of the solution of (2.14) in the two situations of Lemma 2.9 and Lemma 2.10.

Lemma 2.9 Given a polynomial function $w: \mathbb{R} \times \mathbb{R} \rightarrow \mathbb{R}, c_{1} \in \mathbb{R}$ and $c_{2} \in \mathbb{R}$, there exists a unique polynomial solution of

$$
\partial_{t} \varphi(t, \tau)+\partial_{\tau} \varphi(t, \tau)=w(t, \tau), \quad \varphi(0,0)=c_{1} \quad \text { or } \quad \varphi(0,+\infty)=c_{2}
$$

Proof: Writing $w(t, \tau)=\sum_{k=0}^{j} e^{-k \tau} W_{k}(t)$ and $\varphi(t, \tau)=\sum_{k=0}^{r} e^{-k \tau} \varphi_{k}(t)$, the differential equation becomes

$$
\sum_{k=0}^{r} e^{-k \tau}\left(\varphi_{k}^{\prime}(t)-k \varphi_{k}(t)\right)=\sum_{k=0}^{j} e^{-k \tau} W_{k}(t) .
$$

For $k=1, \ldots, j$, there exists a unique polynomial solution of $\varphi_{k}^{\prime}-k \varphi_{k}=W_{k}$, while for $k>j$, $\varphi_{k}^{\prime}-k \varphi_{k}=0$ implies $\varphi_{k}=0$. As for $k=0$, we get $\varphi_{0}(t)=\int_{0}^{t} W_{0}(s) d s+C$ where $C$ is then uniquely defined by $\varphi(0,0)=\sum_{k=1}^{n} \varphi_{k}(0)+C=c_{1}$ or by $\varphi(0,+\infty)=C=c_{2}$.

Lemma 2.10 Given a polynomial function $w: \mathbb{R} \times \mathbb{R} \rightarrow \mathbb{R}$ and $c \in \mathbb{R}$, there exists a unique solution $\varphi(t, \tau)=e^{\tau} \psi(t, \tau)$, with $\psi$ a polynomial function, of :

$$
\partial_{t} \varphi(t, \tau)+\partial_{\tau} \varphi(t, \tau)=e^{\tau} w(t, \tau), \quad \varphi(0,0)=c
$$

Proof: Writing $w(t, \tau)=\sum_{k=0}^{j} e^{-k \tau} W_{k}(t)$ and $\varphi(t, \tau)=e^{\tau} \sum_{k=0}^{r} e^{-k \tau} \varphi_{k}(t)$, the differential equation becomes

$$
\sum_{k=0}^{r} e^{(1-k) \tau}\left(\varphi_{k}^{\prime}(t)+(1-k) \varphi_{k}(t)\right)=\sum_{k=0}^{j} e^{(1-k) \tau} W_{k}(t) .
$$

For $k=0,2, \ldots, j$, there exists a unique polynomial solution of $\varphi_{k}^{\prime}+(1-k) \varphi_{k}=W_{k}$, while for $k>j, \varphi_{k}^{\prime}-k \varphi_{k}=0$ implies $\varphi_{k}=0$. As for $k=1$, we get $\varphi_{1}(t)=\int_{0}^{t} W_{1}(s) \mathrm{ds}+C$, where $C$ is then uniquely defined by $\varphi(0,0)=\varphi_{0}(0)+C+\sum_{k=2}^{n} \varphi_{k}(0)=c$.

Proposition 2.11 1. There exists a unique polynomial map $\gamma \in \mathcal{G}^{\mathbb{R} \times \mathbb{R}}$ solution of (2.14) satisfying $\gamma(0,0)=\mathbb{1}$.

2. There exists a unique polynomial map $\delta \in \mathcal{G}^{\mathbb{R} \times \mathbb{R}}$ solution of

$$
\partial_{t} \delta(t, \tau)+\partial_{\tau} \delta(t, \tau)=\delta(t, \tau) * \beta(\tau)
$$

satisfying both conditions $\left.\delta\right|_{\overline{\mathcal{T}}_{\bullet}}(0,+\infty)=\left.\mathbb{1}\right|_{\overline{\mathcal{T}}_{\bullet}}$ and $\left.\delta\right|_{\overline{\mathcal{T}}_{\circ}}(0,0)=\left.\mathbb{1}\right|_{\overline{\mathcal{T}}_{\circ}}$

Proof: Both assertions can be proved along the same lines and we thus concentrate on the second one. The proof proceeds by induction on the order of trees. For $u=\bullet_{k} \in \mathcal{T}_{\bullet}, k \geq 0$, equation (2.15) with the considered initial conditions gives

$$
\partial_{t} \delta_{\bullet_{k}}(t, \tau)+\partial_{\tau} \delta_{\bullet_{k}}(t, \tau)=e^{-k \tau} \quad \text { and } \quad \lim _{\tau \rightarrow+\infty} \delta_{\bullet_{k}}(t, \tau)=0
$$


which, owing to Lemma 2.9, has a unique polynomial solution. For $v=\circ_{k} \in \mathcal{T}_{\text {o }}, k \geq 0$, we can conclude similarly by Lemma 2.10. Now, consider $u=\left[u_{1}, \ldots, u_{p}, v_{1}, \ldots, v_{q}\right]_{\bullet_{k}} \in \mathcal{T}_{\bullet}$ a tree of order $n \geq 2$. As $\delta$ is a solution of (2.14), we have

$$
\partial_{t} \delta_{u}(t, \tau)+\partial_{\tau} \delta_{u}(t, \tau)=(\delta(t, \tau) * \beta(\tau))_{u}=e^{-(k-q) \tau} \prod_{i=1}^{p} \delta_{u_{i}}(t, \tau) \prod_{j=1}^{q}\left(e^{-\tau} \delta_{v_{j}}(t, \tau)\right):=w(t, \tau)
$$

where, by induction hypothesis, $\delta_{u_{i}}(t, \tau)$ and $e^{-\tau} \delta_{v_{j}}(t, \tau)$ are polynomial, so that $w(t, \tau)$ is also a polynomial function. The assumption on the initial conditions and Lemma 2.9 thus imply that $\delta_{u}(t, \tau)$ is uniquely defined. For a tree $v=\left[u_{1}, \ldots, u_{p}, v_{1}, \ldots, v_{q}\right]_{\circ_{k}} \in \mathcal{T}_{\circ}$ of order $n \geq 2$, we can conclude similarly using Lemma 2.10 .

As we shall see below, the map $\delta$ embeds the dynamics on the center manifold. Its values for trees or orders less than or equal to 2 are given in tables 5 and 6.

\begin{tabular}{|c|c|c|c|c|}
\hline$u$ & $\bullet_{0}$ & $\bullet_{k}$ & $\mathscr{6}_{0}^{0}$ & $\dot{\varphi}_{0}^{r} \quad r \geq 1$ \\
\hline$\delta_{u}$ & $t$ & $\frac{-e^{-k \tau}}{k}$ & $\frac{t^{2}}{2}$ & $\frac{e^{-r \tau}}{r^{2}}$ \\
\hline$u$ & ${ }^{6_{\mathrm{k}}^{\mathrm{r}}} r \geq 1$ & $\delta_{1}^{0}$ & $\int_{k}^{1}$ & 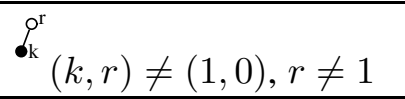 \\
\hline$\delta_{u}$ & $\frac{e^{-(r+k) \tau}}{r(r+k)}$ & $t+e^{-\tau}$ & $\frac{-e^{-k \tau}(1+k t)}{k^{2}}$ & $\frac{-(k+r-1) e^{-k \tau}+k e^{-(k+r-1) \tau}}{(r-1) k(k+r-1)}$ \\
\hline
\end{tabular}

Table 5: Coefficients $\delta$ for trees $u \in \mathcal{T}_{\bullet}$ with $|u| \leq 2$ and $k \geq 1$.

\begin{tabular}{|c|c|c|c|c|c|c|}
\hline$v$ & $\mathrm{o}_{1}$ & $o_{k}$ & $\dot{b}_{1}^{0}$ & $\sigma_{1}^{\sigma^{r}} r \neq 0$ & $\stackrel{\circ}{\mathrm{k}}^{\mathrm{r}} r \neq 0$ & $\dot{d}_{0}^{1}$ \\
\hline$\delta_{v}$ & $t$ & $\frac{1-e^{-k^{\prime} \tau}}{k^{\prime}}$ & $\frac{t^{2}}{2}$ & $\frac{-1+e^{-r \tau}}{r^{2}}$ & $\frac{-1+e^{-\left(k^{\prime}+r\right) \tau}}{r\left(k^{\prime}+r\right)}$ & $-1+e^{\tau}-t$ \\
\hline$\overline{\bar{v}}$ & $\rho_{1}^{1}$ & $\begin{array}{ll}\rho_{1}^{r} & \\
p_{1} & r \neq 1\end{array}$ & $\delta_{2}^{0}$ & $\begin{array}{l}\delta_{\mathrm{k}}^{1} \\
\end{array}$ & ${\stackrel{\rho_{\mathrm{k}}^{\mathrm{r}}}{r}}^{\mathrm{r}} \neq 1, k \geq 2, k+r \neq 2$ & \\
\hline$\delta_{v}$ & $\frac{t^{2}}{2}$ & $\frac{-1+r^{\prime} t+e^{-r^{\prime} \tau}}{\left(r^{\prime}\right)^{2}}$ & $t-1+e^{-\tau}$ & $\frac{1-\left(1+k^{\prime} t\right) e^{-k^{\prime} \tau}}{\left(k^{\prime}\right)^{2}}$ & $\frac{r^{\prime}-\left(k^{\prime}+r^{\prime}\right) e^{-k^{\prime} \tau}+k^{\prime} e^{-\left(k^{\prime}+r^{\prime}\right) \tau}}{r^{\prime} k^{\prime}\left(k^{\prime}+r^{\prime}\right)}$ & \\
\hline
\end{tabular}

Table 6: Coefficients $\delta$ for trees $v$ of $\mathcal{T}_{\circ}$ with $|v| \leq 2, k \neq 1, k^{\prime}=k-1$ and $r^{\prime}=r-1$.

Prior to the next proposition, which states the main result of this subsection, we introduce the following key change of variables of the center-manifold theory.

Definition 2.12 Let $\tilde{\gamma}: \mathbb{R} \rightarrow \mathcal{G}$ be defined by

$$
\left.\tilde{\gamma}\right|_{\overline{\mathcal{T}}_{\bullet}}(t)=\left.\gamma\right|_{\overline{\mathcal{T}}_{\bullet}}(t,+\infty) \quad \text { and }\left.\tilde{\gamma}\right|_{\overline{\mathcal{T}}_{\mathrm{o}}}(t)=\left.\gamma\right|_{\overline{\mathcal{T}}_{\mathrm{o}}}(t, 0) \text {. }
$$

We denote by $\Phi_{0}$ the map $(x, y) \mapsto \Phi_{0}(x, y):=B\left(\tilde{\gamma}^{-1}(0),(x, y)\right)$.

Note that by definition, $\left.\tilde{\gamma}\right|_{\overline{\mathcal{T}}_{\circ}}(0)=\left.\mathbb{1}\right|_{\overline{\mathcal{T}}_{\mathrm{o}}}$, so that $y$ is left unchanged by $\Phi_{0}^{-1}$. The values of $\left.\tilde{\gamma}\right|_{\mathcal{T}_{\bullet}}(0)$ for trees or order less or equal to 2 are given in Table 7 .

Remark 2.13 Denoting $x(t, \tau)=B \bullet\left(\gamma(t, \tau),\left(x_{0}, y_{0}\right)\right)$, the solution $x(t,+\infty)$ in the limit $\tau \rightarrow+\infty$ lies on the center manifold and $x(0,+\infty)$ is nothing but the modified initial condition $x_{0}^{\varepsilon}$. In other words, $\left(x_{0}^{\varepsilon}, y_{0}\right)=\Phi_{0}^{-1}\left(x_{0}, y_{0}\right)$. 


\begin{tabular}{|c|c|c|c|c|}
\hline$u$ & $\bullet_{0}$ & $\bullet_{k}$ & $\mathscr{\delta}_{0}^{0}$ & $\mathscr{6}_{0}^{r} \quad r \geq 1$ \\
\hline$\gamma_{u}(0,+\infty)$ & 0 & $\frac{1}{k}$ & 0 & $\frac{-1}{r^{2}}$ \\
\hline $\bar{u}$ & $\dot{\phi}^{\mathrm{r}} r \geq 1$ & $\delta_{1}^{0}$ & $\delta_{k}^{1}$ & 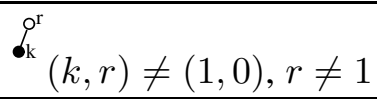 \\
\hline$\gamma_{u}(0,+\infty)$ & $\frac{1}{k(r+k)}$ & -1 & $\frac{1}{k^{2}}$ & $\frac{1}{k(k+r-1)}$ \\
\hline
\end{tabular}

Table 7: Coefficients $\tilde{\gamma}(0)$ for trees $u \in \mathcal{T}_{\bullet}$ with $|u| \leq 2$ and $k \geq 1$.

Proposition 2.14 The following relations hold true for all $\left(t, t^{\prime}, \tau\right) \in \mathbb{R} \times \mathbb{R} \times \mathbb{R}$ :

$$
\begin{aligned}
\gamma\left(t+t^{\prime}, \tau\right) & =\tilde{\gamma}\left(t^{\prime}\right) * \tilde{\gamma}^{-1}(0) * \gamma(t, \tau), \\
\gamma(t, \tau) & =\tilde{\gamma}(t) * \tilde{\gamma}^{-1}(0) * \gamma(0, \tau), \\
\tilde{\gamma}\left(t+t^{\prime}\right) & =\tilde{\gamma}\left(t^{\prime}\right) * \tilde{\gamma}^{-1}(0) * \tilde{\gamma}(t), \\
\gamma\left(t+t^{\prime}, \tau\right) & =\gamma\left(t^{\prime}, 0\right) * \gamma(t, \tau), \\
\gamma(t, \tau) & =\gamma(t, 0) * \gamma(0, \tau), \\
\gamma\left(t+t^{\prime}, 0\right) & =\gamma\left(t^{\prime}, 0\right) * \gamma(t, 0) .
\end{aligned}
$$

Proof: Premultiplying (2.14) by $\tilde{\gamma}^{-1}\left(t^{\prime}\right)$ and using the right-linearity of the $*$-product, one sees that $\tilde{\gamma}^{-1}\left(t^{\prime}\right) * \gamma\left(t+t^{\prime}, \tau\right)$ also satisfies an equation of the form (2.15) with

$$
\left.\left(\tilde{\gamma}^{-1}\left(t^{\prime}\right) * \gamma\left(t^{\prime},+\infty\right)\right)\right|_{\overline{\mathcal{T}}_{\bullet}}=\left.\tilde{\gamma}^{-1}\left(t^{\prime}\right) * \gamma\right|_{\overline{\mathcal{T}}_{\bullet}}\left(t^{\prime},+\infty\right)=\mathbb{1}_{\overline{\mathcal{T}}}
$$

and

$$
\left.\left(\tilde{\gamma}^{-1}\left(t^{\prime}\right) * \gamma\left(t^{\prime}, 0\right)\right)\right|_{\overline{\mathcal{T}}_{\circ}}=\left.\tilde{\gamma}^{-1}\left(t^{\prime}\right) * \gamma\right|_{\overline{\mathcal{T}}_{\circ}}\left(t^{\prime}, 0\right)=\mathbb{1}_{\overline{\mathcal{T}}_{\circ}} .
$$

By Proposition 2.11, the solution is unique and is thus independent of $t^{\prime}$. In particular, one has

$$
\tilde{\gamma}^{-1}\left(t^{\prime}\right) * \gamma\left(t+t^{\prime}, \tau\right)=\tilde{\gamma}^{-1}(0) * \gamma(t, \tau)=\delta(t, \tau)
$$

which proves (2.16). Equation (2.17) is then obtained from (2.16) by taking $t=0$ and renaming $t^{\prime}$ by $t$ while (2.18) follows from (2.16) by putting $\tau=+\infty$ for trees in $\mathcal{T}_{\bullet}$ and $\tau=0$ for trees in $\mathcal{T}_{\circ}$. The last three equations are obtained similarly by noticing that $\gamma^{-1}\left(t^{\prime}, 0\right) * \gamma\left(t+t^{\prime}, \tau\right)$ also satisfies equation (2.14) with initial condition $\gamma^{-1}\left(t^{\prime}, 0\right) * \gamma\left(t^{\prime}, 0\right)=\mathbb{1}$ and invoking again Proposition 2.11.

We emphasize that, in passing, we have obtained (see (2.22) the relation $\delta(t, \tau)=\tilde{\gamma}^{-1}(0) * \gamma(t, \tau)$. Hence, the solution of the original problme can be recovered from $\delta(t, t)$ simply by starting from the modified initial condition

$$
B\left(\delta(t, t), \Phi_{0}^{-1}\left(x_{0}, y_{0}\right)\right)=B\left(\gamma(t, t),\left(x_{0}, y_{0}\right)\right),
$$

where $\Phi_{0}$ has been introduced in Definition 2.12.

\subsection{Dynamics on the center manifold}

The map $\delta$, being a polynomial map, can be decomposed as

$$
\left.\delta\right|_{\mathcal{T}_{\bullet}}(t, \tau)=\left.\delta^{\infty}\right|_{\mathcal{T}_{\bullet}}(t)+\left.e^{-\tau} \delta^{R}\right|_{\mathcal{T}_{\bullet}}(t, \tau) \quad \text { and }\left.\delta\right|_{\mathcal{T}_{\circ}}(t, \tau)=\left.e^{\tau} \delta^{\infty}\right|_{\mathcal{T}_{\circ}}(t)+\left.\delta^{R}\right|_{\mathcal{T}_{\circ}}(t, \tau)
$$


Definition 2.15 The norm $\|\cdot\|$ is defined recursively on $\mathcal{T}$ as follows: for all $k \in \mathbb{N},\left\|\bullet_{k}\right\|=\left\|\circ_{k}\right\|=k$ and for $w=\left[u_{1}, \ldots, u_{p}, v_{1}, \ldots, v_{q}\right]_{\bullet_{k}}$ or $w=\left[u_{1}, \ldots, u_{p}, v_{1}, \ldots, v_{q}\right]_{\circ_{k}}$, then

$$
\|w\|=k-q+\sum_{i=1}^{p}\left\|u_{i}\right\|+\sum_{i=1}^{q}\left\|v_{i}\right\| .
$$

Remark 2.16 Trees with zero norm are distinctive for the corresponding elementary differentials do not depend on $y$. This stems from the very definition of $f_{k}(x, y)$ and $g_{k}(x, y)$ as $k$-linear maps w.r.t. the variable $y$, and from the fact that, in a tree with zero norm, all functions $f_{k}$ or $g_{k}$ appearing are differentiated exactly $k$ times. It is also worth mentioning that in a tree of zero norm, the index carried by any vertex is exactly equal to the number of upcoming $\circ$ branches. In this sense, the set of zero norm indexed partitioned trees is isomorphic to the set of partitioned trees with no label. For the sake of illustration, consider for instance the zero-norm tree $u=\left[\bullet_{0}, \bullet_{0}, \circ_{0}, \circ_{0}, \circ_{0}\right] \bullet_{3}$. Since $\partial_{y}^{3} f_{3}(x, y)=\frac{1}{3 !} \partial_{y}^{3}\left(\left(\partial_{y}^{3} f\right)(x, 0) y^{3}\right)=\left(\partial_{y}^{3} f\right)(x, 0)$, we have

$$
\begin{aligned}
\mathcal{F}_{u}(x, y) & =\left(\partial_{x}^{2} \partial_{y}^{3} f\right)(x, 0)\left(\mathcal{F}_{\bullet_{0}}(x, y), \mathcal{F}_{\bullet_{0}}(x, y), \mathcal{F}_{\circ_{0}}(x, y), \mathcal{F}_{\mathrm{o}_{0}}(x, y), \mathcal{F}_{\circ_{0}}(x, y)\right) \\
& =\left(\partial_{x}^{2} \partial_{y}^{3} f\right)(x, 0)(f(x, 0), f(x, 0), g(x, 0), g(x, 0), g(x, 0)) .
\end{aligned}
$$

Proposition 2.17 For all $u=\left[u_{1}, \ldots, u_{p}, v_{1}, \ldots, v_{q}\right]_{\bullet_{k}} \in \mathcal{T}_{\bullet}$ and all $v=\left[u_{1}, \ldots, u_{p}, v_{1}, \ldots, v_{q}\right]_{\circ_{k}} \in$ $\mathcal{T}_{\circ}$ such that $q=k$, the following relations

$$
\begin{aligned}
& \dot{\delta}_{u}^{\infty}(t)=\prod_{i=1}^{p} \delta_{u_{i}}^{\infty}(t) \prod_{j=1}^{q} \delta_{v_{j}}^{\infty}(t), \quad \delta_{u}^{\infty}(0)=0 \\
& \text { and } \quad \dot{\delta}_{v}^{\infty}(t)+\delta_{v}^{\infty}(t)=\prod_{i=1}^{p} \delta_{u_{i}}^{\infty}(t) \prod_{j=1}^{q} \delta_{v_{j}}^{\infty}(t)
\end{aligned}
$$

are satisfied. Furthermore, if $w \in \mathcal{T}$ is such that $\|w\|>0$, then $\delta_{w}^{\infty}(t)=0$ for all $t \in \mathbb{R}$.

Proof: By definition of $\delta$, we have $\left(\partial_{t}+\partial_{\tau}\right) \delta(t, \tau)=\delta(t, \tau) * \beta(\tau)$ with $\left.\delta^{\infty}\right|_{\mathcal{T}_{\bullet}}(0)=\left.\delta\right|_{\mathcal{T}_{\bullet}}(0,+\infty)=$ $\left.\mathbb{1}\right|_{\mathcal{T}_{\bullet}}$. Hence, for all $u=\left[u_{1}, \ldots, u_{p}, v_{1}, \ldots, v_{q}\right]_{\bullet_{k}} \in \mathcal{T}_{\bullet}$, one has

$$
e^{-(k-q) \tau} \prod_{i=1}^{p} \delta_{u_{i}}^{\infty}(t) \prod_{j=1}^{q} \delta_{v_{j}}^{\infty}(t)+e^{-\tau} w(t, \tau)=\dot{\delta}_{u}^{\infty}(t)+e^{-\tau} \tilde{w}(t, \tau)
$$

where $w$ and $\tilde{w}$ are polynomial functions. Similarly, for all $v=\left[u_{1}, \ldots, u_{p}, v_{1}, \ldots, v_{q}\right]_{\circ_{k}} \in \mathcal{T}_{\circ}$, one has

$$
e^{-(k-1-q) \tau} \prod_{i=1}^{p} \delta_{u_{i}}^{\infty}(t) \prod_{j=1}^{q} \delta_{v_{j}}^{\infty}(t)+w(t, \tau)=e^{\tau}\left(\dot{\delta}_{v}^{\infty}(t)+\delta_{v}^{\infty}(t)\right)+\tilde{w}(t, \tau) .
$$

For $k=q$, this clearly proves equations (2.24).

Now, suppose that $\|u\|>0$ and $\|v\|>0$ respectively: then either $k<q$ or $k=q$ and at least one amongst the branches $u_{i}$ or $v_{j}$ has a strictly positive norm. In the first case, we obtain respectively 
$\dot{\delta}_{u}^{\infty}(t)=0$ and $\dot{\delta}_{v}^{\infty}(t)+\delta_{v}^{\infty}(t)=0$ (according to (2.25) and (2.26)), so that $\delta_{u}^{\infty}(t)=0$ owing to the initial condition $\delta_{u}^{\infty}(0)=0$, and $\delta_{v}^{\infty}(t)=0$ owing to the fact that $\delta_{v}^{\infty}(t)$ is polynomial in $t$. In the second case, relation (2.24) is satisfied with a right-hand side that vanishes owing to an induction argument, and we can conclude similarly.

Remark 2.18 Note that the initial condition $\left.\delta^{\infty}\right|_{\mathcal{T}_{\circ}}(0)$ is not known a priori. It is actually determined by solving equation (2.24). This is accordance with the fact that $\delta^{\infty}$ are the B-series coefficients of the asymptotic dynamics of $(x(t), z(t))$, the solution of $(2.1)$.

Relation (2.16) can be rewritten as

$$
\delta\left(t+t^{\prime}, \tau\right)=\tilde{\gamma}^{-1}(0) * \tilde{\gamma}\left(t^{\prime}\right) * \delta(t, \tau) .
$$

Furthermore, it is clear that

$$
\left.\delta^{\infty}\right|_{\mathcal{T}_{\bullet}}(t)=\left.\delta\right|_{\mathcal{T}_{\bullet}}(t,+\infty)=\left.\tilde{\gamma}^{-1}(0) * \gamma\right|_{\mathcal{T}_{\bullet}}(t, \infty)=\left.\tilde{\gamma}^{-1}(0) * \tilde{\gamma}\right|_{\mathcal{T}_{\bullet}}(t),
$$

and that

$$
\left.\delta^{\infty}\right|_{\mathcal{T}_{\circ}}(t)=\left.\lim _{\tau \rightarrow \infty} e^{-\tau} \delta\right|_{\mathcal{T}_{\circ}}(t, \tau)
$$

Passing to the appropriate limits, we thus obtain

$$
\delta^{\infty}\left(t+t^{\prime}\right)=\left(\tilde{\gamma}^{-1}(0) * \tilde{\gamma}\left(t^{\prime}\right)\right) * \delta^{\infty}(t) .
$$

Given now that $\delta_{w}^{\infty} \equiv 0$ if $\|w\|>0$, the left term in the star product of the right-hand side is evaluated only for trees of $\mathcal{T}_{\bullet}$, and since

$$
\left.\left(\tilde{\gamma}^{-1}(0) * \tilde{\gamma}\left(t^{\prime}\right)\right)\right|_{\mathcal{T}_{\bullet}}=\left.\tilde{\gamma}^{-1}(0) * \tilde{\gamma}\right|_{\mathcal{T}_{\bullet}}\left(t^{\prime}\right)=\left.\delta^{\infty}\right|_{\mathcal{T}_{\bullet}}\left(t^{\prime}\right)
$$

we may write

$$
\delta^{\infty}\left(t+t^{\prime}\right)=\delta^{\infty}\left(t^{\prime}\right) * \delta^{\infty}(t)
$$

or

$$
\delta^{\infty}\left(t+t^{\prime}\right)=\left.\delta^{\infty}\right|_{\mathcal{T}_{\bullet}}\left(t^{\prime}\right) * \delta^{\infty}(t)
$$

at the price of a slight abuse of notations (we have indeed here $\delta_{\emptyset_{y}}^{\infty}\left(t^{\prime}\right)=0$, in contrast with the definition of the $*$-product in Subsection 2.3). The perfectly rigorous way to write (2.27) would be

$$
\delta^{\infty}\left(t+t^{\prime}\right)=\left(\mathbb{1}^{z}+\delta^{\infty}\left(t^{\prime}\right)\right) * \delta^{\infty}(t)
$$

where $\mathbb{1}_{w}^{z}=0$ for all $w \notin \overline{\mathcal{T}}_{\bullet} \cup \mathcal{T}_{\circ}$ and $\mathbb{1}_{\emptyset_{y}}^{z}=1$, where the addition of the term $\mathbb{1}^{z}$ is harmless and only technical, since it does not appear in the effective computations of the star product.

Remark 2.19 Note that the B-series $B\left(\delta^{\infty}(t),(x, y)\right)$ is of the form $(x+\mathcal{O}(\varepsilon), \mathcal{O}(\varepsilon))$, since for all $t \geq 0, \delta_{\emptyset_{z}}^{\infty}(t)=0$. Besides, since $\delta_{w}^{\infty} \equiv 0$ for $\|w\|>0$, that it does not depend on $z$, that is to say

$$
B\left(\delta^{\infty}(t),(x, y)\right)=B\left(\delta^{\infty}(t),(x, 0)\right) .
$$

As a consequence, the asymptotic behaviour depends on $z_{0}=y_{0}$ only through the modified intial condition $x_{0}^{\varepsilon}$. 
In the sequel, we shall denote

$$
\left(x^{\infty}(t), z^{\infty}(t)\right)=B\left(\delta^{\infty}(t),\left(x_{0}^{\varepsilon}, 0\right)\right),
$$

where $\left(x_{0}^{\varepsilon}, y_{0}\right)=\Phi_{0}^{-1}\left(x_{0}, y_{0}\right)$. It may be seen as the trajectory

$$
(x(t, \tau), z(t, \tau))=\left(B \bullet\left(\delta(t, \tau),\left(x_{0}^{\varepsilon}, z_{0}\right)\right), B_{\circ}\left(e^{-\tau} \delta(t, \tau),\left(x_{0}^{\varepsilon}, z_{0}\right)\right)\right)
$$

in the limit $\tau=+\infty$, i.e. as the shadow solution of $(x(t), y(t))$ on the center manifold. We are now in position to identify the center-manifold function $\varepsilon h$ and the vector field $\varepsilon F$ of the slow dynamics in the variable $x$.

Proposition 2.20 Consider the two functions $h$ and $F$ defined by their B-series expansions

$$
\varepsilon h(x):=B_{\circ}\left(\delta^{\infty}(0),(x, 0)\right) \text { and } \varepsilon F(x):=B_{\bullet}\left(\dot{\delta}^{\infty}(0),(x, 0)\right) .
$$

The following relations hold true

$$
\left.\varepsilon f(x, \varepsilon h(x))=B \bullet\left(\dot{\delta}^{\infty}(0),(x, 0)\right) \text { and } \varepsilon g(x, \varepsilon h(x))\right)=B_{\circ}\left(\dot{\delta}^{\infty}(0)+\delta^{\infty}(0),(x, 0)\right) .
$$

In particular, one has $F(x)=f(x, \varepsilon h(x))$.

Proof: By definition of $\beta$, we have $(\varepsilon f(x, z), \varepsilon g(x, z))=B(\beta(0),(x, z))$. Besides, we can write

$$
(x, \varepsilon h(x))=B\left(\delta^{\infty}(0),(x, 0)\right)=B\left(\mathbb{1}^{z}+\delta^{\infty}(0),(x, 0)\right),
$$

so that, using the star-product, we get

$$
(\varepsilon f(x, \varepsilon h(x)), \varepsilon g(x, \varepsilon h(x)))=B\left(\left(\mathbb{1}^{z}+\delta^{\infty}(0)\right) * \beta(0),(x, 0)\right) .
$$

To sum up, we wish to prove that

$$
\begin{aligned}
& B_{\bullet}\left(\left(\mathbb{1}^{z}+\delta^{\infty}(0)\right) * \beta(0),(x, 0)\right)=B_{\bullet}\left(\dot{\delta}^{\infty}(0),(x, 0)\right), \\
& B_{\circ}\left(\left(\mathbb{1}^{z}+\delta^{\infty}(0)\right) * \beta(0),(x, 0)\right)=B_{\circ}\left(\dot{\delta}^{\infty}(0)+\delta^{\infty}(0),(x, 0)\right) .
\end{aligned}
$$

Now, given that $\left.\mathcal{F}_{w}(x, z)\right|_{z=0}=0$ whenever $\|w\|>0$, all we have to prove is that for all $w \in \mathcal{T}$ with $\|w\|=0$, one has

$$
\begin{aligned}
& \left(\left(\mathbb{1}^{z}+\delta^{\infty}(0)\right) * \beta(0)\right)_{w}=\dot{\delta}_{w}^{\infty}(0) \text { if } w \in \mathcal{T}_{\bullet}, \\
& \left(\left(\mathbb{1}^{z}+\delta^{\infty}(0)\right) * \beta(0)\right)_{w}=\dot{\delta}_{w}^{\infty}(0)+\delta_{w}^{\infty}(0) \text { if } w \in \mathcal{T}_{\circ}
\end{aligned}
$$

Consider a tree $w \in \mathcal{T}$ either of the form $\left[u_{1}, \ldots, u_{p}, v_{1}, \ldots, v_{q}\right]_{\bullet_{q}}$ or $\left[u_{1}, \ldots, u_{p}, v_{1}, \ldots, v_{q}\right]_{\circ_{q}}$. According to Proposition 2.17 on the one hand, and by definition of the $*$-product on the other hand, we indeed have

$$
\begin{array}{r}
\dot{\delta}_{w}^{\infty}(0)=\prod_{i=1}^{p} \delta_{u_{i}}^{\infty}(0) \prod_{j=1}^{q} \delta_{v_{j}}^{\infty}(0)=\left[\left(\mathbb{1}^{z}+\delta^{\infty}(0)\right) * \beta(0)\right]_{w} \text { if } w \in \mathcal{T}_{\bullet}, \\
\dot{\delta}_{w}^{\infty}(0)+\delta_{w}^{\infty}(0)=\prod_{i=1}^{p} \delta_{u_{i}}^{\infty}(0) \prod_{j=1}^{q} \delta_{v_{j}}^{\infty}(0)=\left[\left(\mathbb{1}^{z}+\delta^{\infty}(0)\right) * \beta(0)\right]_{w} \text { if } w \in \mathcal{T}_{\circ},
\end{array}
$$

where we used the explicit definition of $\beta$ (as in (2.10)). 
Theorem 2.21 The solution $\left(x^{\infty}(t), z^{\infty}(t)\right)$ defined in (2.29) satisfies the following center-manifold system

$$
\left\{\begin{array}{l}
\dot{x}^{\infty}(t)=\varepsilon F\left(x^{\infty}(t)\right)=\varepsilon f\left(x^{\infty}(t), \varepsilon h\left(x^{\infty}(t)\right)\right) \\
z^{\infty}(t)=\varepsilon h\left(x^{\infty}(t)\right)
\end{array},\right.
$$

with initial condition $\left(x^{\infty}(0), z^{\infty}(0)\right)=\left(x_{0}^{\varepsilon}, \varepsilon h\left(x_{0}^{\varepsilon}\right)\right)$.

Proof: For trees of $\mathcal{T}_{\circ}$, relation (2.28) becomes

$$
\left.\delta^{\infty}\right|_{\mathcal{T}_{0}}\left(t+t^{\prime}\right)=\left.\left.\delta^{\infty}\right|_{\mathcal{T}_{\bullet}}(t) * \delta^{\infty}\right|_{\mathcal{T}_{0}}\left(t^{\prime}\right)
$$

so that, by choosing $t^{\prime}=0$, one has

$$
\left.\delta^{\infty}\right|_{\mathcal{T}_{\circ}}(t)=\left.\left.\delta^{\infty}\right|_{\mathcal{T}_{\bullet}}(t) * \delta^{\infty}\right|_{\mathcal{T}_{\circ}}(0),
$$

which proves the second part of (2.31).

As for the first part of the statement, we consider equation (2.28) for trees of $\mathcal{T}_{\bullet}$, differentiate it w.r.t. $t^{\prime}$, then evaluate at $t^{\prime}=0$ and obtain

$$
\left.\dot{\delta}^{\infty}\right|_{\mathcal{T}_{\bullet}}(t)=\left.\left.\delta^{\infty}\right|_{\mathcal{T}_{\bullet}}(t) * \dot{\delta}^{\infty}\right|_{\mathcal{T}_{\bullet}}(0)
$$

\begin{tabular}{|c|c|c|c|c|c|c|c|c|c|}
\hline$\circ_{0}$ & $\mathscr{\mathscr { b }}_{0}^{0}$ & $q_{1}^{0}$ & ${ }_{0}^{\circ}$ & $\begin{array}{l}0^{0} \\
1\end{array}$ & $\begin{array}{l}q_{0}^{0} \\
2\end{array}$ & $\oint_{0}^{0}$ & $\dot{q}_{0}^{0}$ & $\begin{array}{l}q_{0}^{0} \\
\oint_{1}^{1}\end{array}$ & $\begin{array}{l}q_{1}^{0} \\
\delta_{0}\end{array}$ \\
\hline 1 & -1 & 1 & 2 & -1 & 1 & 1 & -2 & 1 & -1 \\
\hline$g$ & $g_{x} f$ & $g_{z} g$ & $g_{x x}(f, f)$ & $g_{x z}(f, g)$ & $g_{z z}(g, g)$ & $g_{x} f_{x} f$ & $g_{z} g_{x} f$ & $g_{z} g_{z} g$ & $g_{x} f_{z} g$ \\
\hline
\end{tabular}

Example 2.22 The first terms of the expansion of $\varepsilon h(x)$ are computed in Table 8, where, for conciseness, we have omitted the argument $(x, 0)$ of all functions and used obvious simplified notations for derivatives. Collecting all terms up to order 3 in $\varepsilon$, we thus obtain

Table 8: Coefficients $\delta^{\infty}(0)$ for trees $u$ of $\mathcal{T}_{\text {。 with }}|u| \leq 3$.

$$
\begin{aligned}
\varepsilon h(x) & =\varepsilon g(x, 0)+\varepsilon^{2}\left(-g_{x}(x, 0) f(x, 0)+g_{z}(x, 0) g(x, 0)\right)+\varepsilon^{3}\left(g_{x x}(x, 0)(f(x, 0), f(x, 0))\right. \\
& +g_{x}(x, 0) f_{x}(x, 0) f(x, 0)-g_{x z}(f(x, 0), g(x, 0))+\frac{1}{2} g_{z z}(x, 0)(g(x, 0), g(x, 0)) \\
& \left.-2 g_{z}(x, 0) g_{x}(x, 0) f(x, 0)+g_{z}(x, 0) g_{z}(x, 0) g(x, 0)-g_{x}(x, 0) f_{z}(x, 0) g(x, 0)\right)+\mathcal{O}\left(\varepsilon^{4}\right) .
\end{aligned}
$$

In next proposition, we prove that the center-manifold function $\varepsilon h$ obtained here satisfies the familiar partial differential equation $(2.32)^{5}$.

Proposition 2.23 The function $h$ satisfies the following partial differential equation

$$
\varepsilon h^{\prime}(x) f(x, \varepsilon h(x))=-h(x)+\varepsilon g(x, \varepsilon h(x)) .
$$

\footnotetext{
${ }^{5}$ This equation is documented in almost every book on the center manifold theorem, see for instance [Car81].
} 


\begin{tabular}{|c|c|c|c|c|c|c|c|c|c|c|}
\hline$u \in \mathcal{T}_{\bullet}$ & $\bullet_{0}$ & $\mathscr{b}_{0}^{0}$ & $\wp_{1}^{0}$ & $\mathfrak{Q}_{0}^{0} 0$ & $\dot{q}_{0}^{0}$ & $\begin{array}{l}q_{0}^{0} \\
0_{1}^{1}\end{array}$ & $q_{0}^{0}$ & $\mathfrak{V}_{0}^{0}$ & $\vartheta^{0}$ & $q_{2}^{0} p^{0}$ \\
\hline$\dot{\delta}^{\infty}(0)$ & 1 & 0 & 1 & 0 & -1 & 1 & 0 & 0 & 0 & 1 \\
\hline$v \in \mathcal{T}_{\circ}$ & $\circ_{0}$ & $\mathscr{b}_{0}^{0}$ & $\rho_{1}^{0}$ & $\mathfrak{Q}_{0}^{0}$ & ${ }_{0}^{0}$ & $\begin{array}{l}0 \\
{ }_{2}^{0}\end{array}$ & $\oint_{0}^{0} 0$ & $q_{0}^{0} 1$ & $\dot{q}_{0}^{0}$ & $\mathfrak{b}_{0}^{0}$ \\
\hline$\dot{\delta}^{\infty}(0)$ & 0 & 1 & 0 & -2 & 1 & 0 & 1 & 0 & 1 & -1 \\
\hline
\end{tabular}

Table 9: Coefficients $\dot{\delta}^{\infty}(0)$ for trees of $\mathcal{T}$ with order $\leq 3$.

Proof: Differentiating $\left(x^{\infty}(t), z^{\infty}(t)\right)=B\left(\delta^{\infty}(t),\left(x_{0}^{\varepsilon}, 0\right)\right)$ w.r.t. $t$, we have $\left(\dot{x}^{\infty}(t), \dot{z}^{\infty}(t)\right)=$ $B\left(\dot{\delta}^{\infty}(t),\left(x_{0}^{\varepsilon}, 0\right)\right)$. From Theorem 2.21, we also have

$$
\left(\dot{x}^{\infty}(t), \dot{z}^{\infty}(t)\right)=\left(\varepsilon F\left(x^{\infty}(t)\right), \varepsilon^{2} h^{\prime}\left(x^{\infty}(t)\right) F\left(x^{\infty}(t)\right)\right)
$$

which, for $t=0$, gives

$$
\varepsilon^{2} h^{\prime}\left(x_{0}^{\varepsilon}\right) F\left(x_{0}^{\varepsilon}\right)=B_{\circ}\left(\dot{\delta}^{\infty}(0),\left(x_{0}^{\varepsilon}, 0\right)\right) .
$$

To sum up, the following three relations hold for all $x$

$$
\begin{array}{ll}
\text { (i) } & \varepsilon^{2} h^{\prime}\left(x_{0}^{\varepsilon}\right) F\left(x_{0}^{\varepsilon}\right)=B_{\circ}\left(\dot{\delta}^{\infty}(0),(x, 0)\right), \\
(\text { ii }) & \varepsilon h(x)=B_{\circ}\left(\delta^{\infty}(0),(x, 0)\right), \\
(\text { iii }) & \varepsilon g(x, \varepsilon h(x))=B_{\circ}\left(\dot{\delta}^{\infty}(0)+\delta^{\infty}(0),(x, 0)\right),
\end{array}
$$

from which we can straightforwardly conclude.

Remark 2.24 Note that if one defines the B-series map $\Pi:(x, z) \mapsto \Pi(x, z):=B\left(\delta^{\infty}(0),(x, z)\right)$ and $\Phi_{0}^{-1}:(x, z) \mapsto \Phi_{0}^{-1}(x, z):=B(\tilde{\gamma}(0),(x, z))$, then the relations

$$
\Pi \circ \Pi=\Pi \quad \text { and } \quad \Phi_{0}^{-1} \circ \Pi=\Pi,
$$

hold true. These two relations guarantee the two consistency requirements that: (i) the B-series $B\left(\delta^{\infty}(0),(x, z)\right)$, mapping $(x, z)$ to $(x, \varepsilon h(x)) \in \mathcal{M}$, is a projection (onto the manifold, perpendicularly to the $x$-axis); (ii) the change of variables $\Phi_{0}$ coincides with the identity map when acting on the manifold $\mathcal{M}$.

The overall results of this subsection are sketched in Figure 1. The main novelty here is the map $\Phi_{0}^{-1}$, whose expansion in powers of $\varepsilon$ we derive, and which tells us how to transform the initial condition.

\subsection{Reduction to normal form}

The center manifold theorem, whether in its standard enunciation 1.1 or in its B-series variation 2.21, decouples the asymptotic dynamics into a slow variable $x$-which obeys a nonstiff reduced modeland an enslaved variable $z$-which becomes a direct function of $x$. In the transient phase (for small values of $t$ ), this remains unsatisfactory since we have no way to recover the full exact solution from the reduced model. The information provided by the initial condition $z_{0}$ is indeed lost as soon as $\Pi$ is 


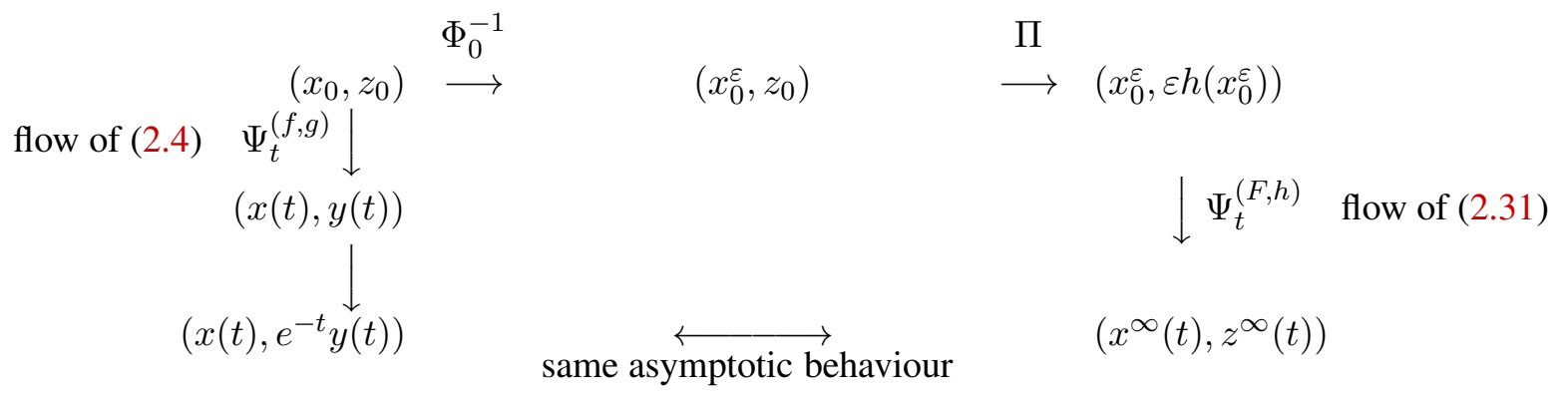

Figure 1: Center-manifold and exact equations.

applied (see Figure 1). If we wish to recover the exact solution of (2.1) (through a decoupled -although not reduced- model), we may further exploit Proposition 2.14. To this aim, we thus consider the map

$$
\tilde{\delta}(t)=\tilde{\gamma}^{-1}(0) * \tilde{\gamma}(t),
$$

which is, roughly speaking, an "unprojected" version of $\delta^{\infty}(t)$. On the one hand, composing (2.17) from the left by $\tilde{\gamma}^{-1}(0)$, we obtain

$$
\delta(t, \tau)=\tilde{\delta}(t) * \delta(0, \tau)=\tilde{\gamma}^{-1}(0) * \gamma(t, \tau)
$$

which means that $\tilde{\delta}(t)$ describes completely the dynamics of the exact solution, characterized by $\gamma(t, t)$, up to changes of variables at initial time and time $t$. As a matter of fact, we have

$$
\gamma(t, t)=\tilde{\gamma}(0) * \tilde{\delta}(t) * \delta(0, t) .
$$

On the other hand, it follows by composing $(2.18)$ by $\tilde{\gamma}^{-1}(0)$ that

$$
\tilde{\delta}\left(t+t^{\prime}\right)=\tilde{\delta}\left(t^{\prime}\right) * \tilde{\delta}(t),
$$

which constitutes a group law for $\tilde{\delta}$. Interestingly enough,

$$
\left.\tilde{\delta}\right|_{\mathcal{T}_{\bullet}}(t)=\left.\lim _{\tau \rightarrow+\infty} \tilde{\gamma}^{-1}(0) * \tilde{\gamma}\right|_{\mathcal{T}_{\bullet}}(t, \tau)=\left.\delta^{\infty}\right|_{\mathcal{T}_{\bullet}}(t),
$$

so that, using Remark 2.19

$$
B_{\bullet}\left(\tilde{\delta}(t),\left(x_{0}^{\varepsilon}, y_{0}\right)\right)=B_{\bullet}\left(\delta^{\infty}(t),\left(x_{0}^{\varepsilon}, y_{0}\right)\right)=B_{\bullet}\left(\delta^{\infty}(t),\left(x_{0}^{\varepsilon}, 0\right)\right)=x^{\infty}(t) .
$$

Theorem 2.25 Let us define the B-series vector field and map

$$
\varepsilon G(x, y)=B_{\circ}(\dot{\tilde{\delta}}(0),(x, y)) \quad \text { and } \quad \Phi_{t}(x, y)=B(\delta(0, t),(x, y)),
$$

and let us denote by $\Psi_{t}^{(F, G)}$ the flow of the differential system

$$
\left\{\begin{array}{ll}
\dot{x}^{\infty} & =\varepsilon F\left(x^{\infty}\right) \\
\dot{\tilde{y}} & =\varepsilon G\left(x^{\infty}, \tilde{y}\right)
\end{array} .\right.
$$

Then the following relation holds true

$$
\Psi_{t}^{(f, g)}\left(x_{0}, y_{0}\right)=\left(\Phi_{t} \circ \Psi_{t}^{(F, G)} \circ \Phi_{0}^{-1}\right)\left(x_{0}, y_{0}\right)
$$


Proof: As already noticed above, we have $B_{\bullet}\left(\tilde{\delta}(t),\left(x_{0}^{\varepsilon}, y_{0}\right)\right)=B_{\bullet}\left(\delta^{\infty}(t),\left(x_{0}^{\varepsilon}, 0\right)\right)=x^{\infty}(t)$, which is known to satisfy the first equation of (2.36). The second equation of (2.36) is obeyed by $\left(x^{\infty}(t), \tilde{y}(t)\right)=$ $B\left(\tilde{\delta}(t),\left(x_{0}^{\varepsilon}, y_{0}\right)\right)$, as can be seen by differentiating equation (2.35) w.r.t. $t$ and evaluating at $t=0$. Now relation (2.37) is just a rewriting of (2.34).

Remark 2.26 The last three relations of Proposition 2.13, namely (2.19), (2.20) and (2.21), are also derived in [MSS16a] (see Example 1 pp. 28 and Theorem 9). They can be used as well to bring the original system to a normal form which however differs from the one obtained here: mutadis mutandis, system (2.36) transforms -via (2.19), (2.20) and (2.21)- into a coupled system in both variables and the gain of working in lower dimension is lost. The corresponding vector field is indeed the B-series with coefficients $\left.\frac{d \gamma(t, 0)}{d t}\right|_{t=0}$ whose first terms can be written as follows

$$
\begin{aligned}
B \cdot( & \left.\left.\frac{d \gamma(t, 0)}{d t}\right|_{t=0},(x, y)\right)=\varepsilon f(x, 0)+\varepsilon^{2} \partial_{y} f(x, 0) g(x, 0) \\
& +\varepsilon^{2} \sum_{k \geq 1} \frac{1}{k !}\left(\frac{1}{k} \partial_{x} f(x, 0) \partial_{y}^{k} f(x, 0) y^{k}-\partial_{z}^{k} f(x, 0)\left(y^{k-1}, \partial_{y} g(x, 0) y\right)\right)+\mathcal{O}\left(\varepsilon^{3}\right)
\end{aligned}
$$

for component $\dot{x}$ and

$$
\begin{gathered}
B_{\circ}\left(\left.\frac{d \gamma(t, 0)}{d t}\right|_{t=0},(x, y)\right)=\varepsilon g(x, 0)+\varepsilon^{2}\left(\frac{1}{2} \partial_{x} \partial_{y}^{2} g(x, 0)\left(y^{2}, f(x, 0)\right)-\partial_{x} g(x, 0) \partial_{y} f(x, 0) y\right) \\
+\varepsilon^{2} \sum_{k \geq 1} \frac{1}{k k !}\left(\partial_{x} \partial_{y} g(x, 0)\left(y, \partial_{y}^{k} f(x, 0) y^{k}\right)-\partial_{y}^{k+1} g(x, 0)\left(y^{k}, \partial_{y} g(x, 0) y\right)\right)+\mathcal{O}\left(\varepsilon^{3}\right)
\end{gathered}
$$

for component $\dot{y}$. These expansions make apparent the dependence w.r.t. both $x$ and $y$ of both compo-

\begin{tabular}{|c|c|c|c|c|}
\hline$u$ & $\bullet_{0}$ & $\bullet_{k}$ & $\mathscr{0}_{0}^{0}$ & $\dot{\sigma}^{r} r \geq 1$ \\
\hline$\delta_{u}(0, t)$ & 0 & $\frac{-e^{-k t}}{k}$ & 0 & $\frac{e^{-r t}}{r^{2}}$ \\
\hline$u$ & ${\overline{\delta_{k}}}_{r \geq 1}^{r}$ & $?_{0}^{0}$ & $\overline{\rho_{0}^{1}}$ & 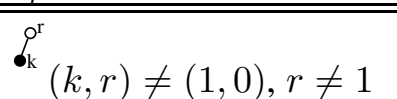 \\
\hline$\delta_{u}(0, t)$ & $\frac{e^{-(r+k) t}}{r(r+k)}$ & 0 & $\frac{-e^{-k t}}{k^{2}}$ & $\frac{-(k+r-1) e^{-k t}+k e^{-(k+r-1) t}}{(r-1) k(k+r-1)}$ \\
\hline
\end{tabular}
nents, in contrast with (2.36), which may be thought of as a triangularization.

In order to impart to Theorem 2.25 its practical value, we list coefficients $\delta(0, t)$, relative to $\Phi_{t}$, for all trees of orders less than or equal to two, in Table 10 and Table 11. We further illustrate Theorems

Table 10: Coefficients $\delta(0, t)$ for trees $u \in \mathcal{T}_{\bullet}$ with $|u| \leq 2$ and $k \geq 1$.

2.21 and 2.25 schematically in Figure 2.

\section{Numerical illustration of the results}

For the sake of illustration, we next compute the various expansions considered in Theorem 2.25 in two simple examples. All expansions are truncared at $\varepsilon^{3}$, i.e. $\varepsilon^{4}$-terms and smaller are neglected. 


\begin{tabular}{|c|c|c|c|c|c|c|}
\hline$v$ & $\mathrm{o}_{1}$ & $\circ_{k}$ & $\dot{d}_{1}^{0}$ & ${\stackrel{\sigma_{1}^{r}}{r}}_{r \neq 0}$ & $\dot{o}_{\mathrm{k}}^{\mathrm{r}} r \neq 0$ & $\dot{b}_{0}^{1}$ \\
\hline$\delta_{v}(0, t)$ & 0 & $\frac{1-e^{-k^{\prime} t}}{k^{\prime}}$ & 0 & $\frac{-1+e^{-r t}}{r^{2}}$ & $\frac{-1+e^{-\left(k^{\prime}+r\right) t}}{r\left(k^{\prime}+r\right)}$ & 0 \\
\hline $\bar{v}$ & $\rho_{1}^{1}$ & 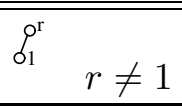 & $\rho_{2}^{0}$ & $\delta_{\mathrm{k}} \quad k \geq 2$ & ${ }^{\rho_{\mathrm{k}}^{\mathrm{r}}} r \neq 1, k \geq 2, k+r \neq 2$ & \\
\hline$\delta_{v}(0, t)$ & 0 & $\frac{-1+e^{-r^{\prime} t}}{\left(r^{\prime}\right)^{2}}$ & $-1+e^{-t}$ & $\frac{1-e^{-k^{\prime} t}}{\left(k^{\prime}\right)^{2}}$ & $\frac{r^{\prime}-\left(k^{\prime}+r^{\prime}\right) e^{-k^{\prime} t}+k^{\prime} e^{-\left(k^{\prime}+r^{\prime}\right) t}}{r^{\prime} k^{\prime}\left(k^{\prime}+r^{\prime}\right)}$ & \\
\hline
\end{tabular}

Table 11: Coefficients $\delta(0, t)$ for trees $v$ of $\mathcal{T}_{\circ}$ with $|v| \leq 2, k \neq 1, k^{\prime}=k-1$ and $r^{\prime}=r-1$.

$$
\begin{aligned}
& \Phi_{0}^{-1} \quad \Pi \\
& \left(x_{0}, y_{0}\right) \quad \longrightarrow \quad\left(x_{0}^{\varepsilon}, y_{0}\right) \quad \longrightarrow\left(x_{0}^{\varepsilon}, \varepsilon h\left(x_{0}^{\varepsilon}\right)\right) \\
& \text { flow of } \downarrow(2.4) \Psi_{t}^{(f, g)} \quad \Psi_{t}^{(F, G)} \text { flow } \downarrow \text { of (2.36) } \\
& \Phi_{t} \\
& (x(t), y(t)) \quad \longleftarrow \quad\left(x^{\infty}(t), \tilde{y}(t)\right) \\
& \downarrow \\
& \left(x(t), e^{-t} y(t)\right) \\
& \text { same asymptotic behaviour } \\
& \Psi_{t}^{(F, h)} \downarrow \text { flow of (2.31) } \\
& \left(x^{\infty}(t), z^{\infty}(t)\right)
\end{aligned}
$$

Figure 2: Center-manifold and normal-form equations.

\subsection{Two coupled scalar equations}

The first system we consider is of the form

$$
\left\{\begin{array}{l}
\dot{x}=-\varepsilon x^{3}\left(z-\frac{z^{3}}{3}\right) \\
\dot{z}=-z+\varepsilon x\left(1-\frac{z^{2}}{2}\right)
\end{array},\right.
$$

with initial conditions $x_{0}=0.8$ and $z_{0}=0.05$. Its exact solution ${ }^{6}$ for $\varepsilon=0.01$ is drawn on the left of Figure 3. Functions $f$ and $g$ being polynoms of respective degrees 3 and 2 in $z$, all functions $f_{k}$ and $g_{k}$ vanish identically, except the following ones:

$$
\begin{array}{llrl}
f_{1}(x, z) & =\partial_{z} f(x, 0) z=-x^{3} z, & f_{3}(x, z) & =\frac{1}{6} \partial_{z}^{3} f(x, 0) z^{3}=\frac{1}{3} x^{3} z^{3}, \\
g_{0}(x, z) & =g(x, 0)=x, & g_{2}(x, z) & =\frac{1}{2} \partial_{z}^{2} g(x, 0) z^{2}=-\frac{1}{2} x z^{2} .
\end{array}
$$

As a result, only ten trees in $\mathcal{T}_{\bullet}$ of orders less than or equal to 2 have to be considered. Their corresponding coefficients $\tilde{\gamma}(0)$ can be read from Table 7 and are listed in Table 12 together with their associated elementary differentials. The second-order truncated expansion of $x_{0}^{\varepsilon}$, defined by $\left(x_{0}^{\varepsilon}, y_{0}\right)=\Phi_{0}^{-1}\left(x_{0}, y_{0}\right)$, is thus of the form $x_{0}+\varepsilon X_{1}+\varepsilon^{2} X_{2}$, with

$$
X_{1}=-x_{0}^{3} y_{0}+\frac{1}{9} x_{0}^{3} y_{0}^{3} \quad \text { and } \quad X_{2}=-\frac{1}{3} x_{0}^{5} y_{0}^{4}+\frac{3}{2} x_{0}^{5} y_{0}^{2}+\frac{1}{54} x_{0}^{5} y_{0}^{6}+x_{0}^{4}+\frac{5}{12} x_{0}^{4} y_{0}^{2}-\frac{1}{24} x_{0}^{4} y_{0}^{4} .
$$

\footnotetext{
${ }^{6}$ It is actually obtained as the result of a very high-precision simulation.
} 

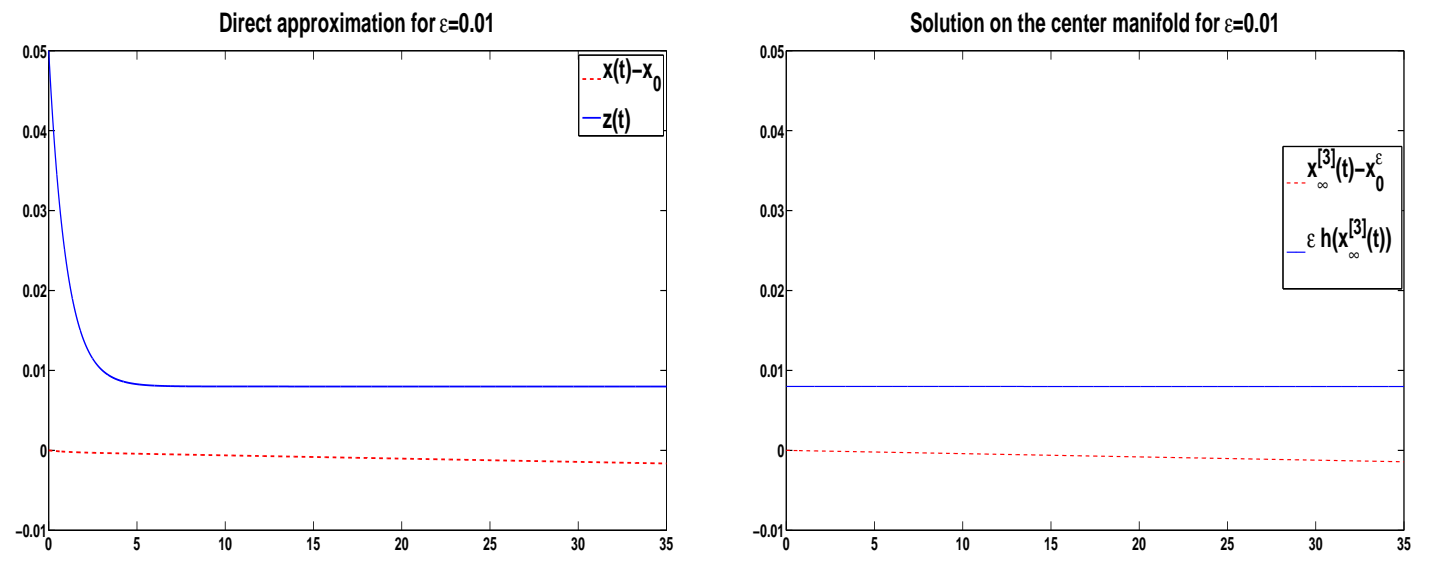

Figure 3: Solution $(x(t), y(t))$ of (3.1) (left) and solution $\left(x_{\infty}^{[3]}(t), z_{\infty}^{[3]}(t)\right)$ of (3.2) (right).

\begin{tabular}{|c|c|c|c|c|c|c|c|c|c|c|}
\hline$u \in \mathcal{T}_{\bullet}$ & $\bullet_{1}$ & $\bullet$ & $0_{1}^{3}$ & $0^{1}$ & $\phi_{1}^{1}$ & $0^{3}$ & $\wp_{0}^{0}$ & $\delta_{1}^{2}$ & $\rho_{6}^{0}$ & $o_{3}^{2}$ \\
\hline$\tilde{\gamma}_{u}(0)$ & 1 & $1 / 3$ & $1 / 4$ & $1 / 12$ & $1 / 2$ & $1 / 18$ & -1 & $1 / 2$ & $1 / 6$ & $1 / 12$ \\
\hline $\mathcal{F}_{u}(x, y)$ & $-x^{3} y$ & $\frac{1}{3} x^{3} y^{3}$ & $-x^{5} y^{4}$ & $-x^{5} y^{4}$ & $3 x^{5} y^{2}$ & $\frac{1}{3} x^{5} y^{6}$ & $x^{4}$ & $\frac{1}{2} x^{4} y^{2}$ & $x^{4} y^{2}$ & $-\frac{1}{2} x^{4} y^{4}$ \\
\hline
\end{tabular}

Table 12: Coefficients $\tilde{\gamma}(0)$ and elementary differentials for trees of $\mathcal{T}_{\bullet}$ with order $\leq 2$.

Getting the third-order term requires a few more calculations that we do not reproduce here:

$$
\begin{aligned}
X_{3}=\left(\frac{5}{6} y_{0}\right. & \left.-\frac{7}{36} y_{0}^{3}+\frac{1}{60} y_{0}^{5}\right) x_{0}^{5}+\left(-\frac{7}{2} y_{0}-\frac{695}{504} y_{0}^{3}+\frac{33}{100} y_{0}^{5}-\frac{1}{60} y_{0}^{7}\right) x_{0}^{6} \\
& +\left(-\frac{5}{2} y_{0}^{3}+\frac{5}{6} y_{0}^{5}-\frac{5}{54} y_{0}^{7}+\frac{5}{1458} y_{0}^{9}\right) x_{0}^{7} .
\end{aligned}
$$

Much fewer terms are present in the expansion of $\varepsilon h(x)$, as coefficients $\delta^{\infty}(0)$ vanish for all trees with a non-zero norm. Truncating once again at order 3 , we indeed obtain

$$
\varepsilon h^{[3]}(x):=\varepsilon x+\varepsilon^{3}\left(-\frac{1}{2} x^{3}+x^{4}\right)=\varepsilon h(x)+\mathcal{O}\left(\varepsilon^{4}\right)
$$

Figure 3 (right) then represents the solution of the following system, which is a third-order approximation of the center-manifold system (2.31)

$$
\left\{\begin{aligned}
\dot{x}_{\infty}^{[3]} & =\varepsilon f\left(x_{\infty}^{[3]}, \varepsilon h^{[3]}\left(x_{\infty}^{[3]}\right)\right) \\
\dot{z}_{\infty}^{[3]} & =\varepsilon h^{[3]}\left(x_{\infty}^{[3]}\right)
\end{aligned}\right.
$$

with initial condition $x_{\infty}^{[3]}(0)=x_{0}^{\varepsilon}$. Figure 4 presents simultaneously $x(t)$ and $x_{\infty}^{[3]}(t)$ (on the left), $z(t)$ and $\varepsilon h\left(x_{\infty}^{[3]}(t)\right)$ (on the right). Finally, the differences $x(t)-x_{\infty}^{[3]}(t)$ (left) and $z(t)-\varepsilon h\left(x_{\infty}^{[3]}(t)\right)$ (right) are drawn on Figure 5 for several values of $\varepsilon$, from which it can be fairly inferred that numerical results are in perfect agreement with the expected error $\mathcal{O}\left(e^{-\mu t}\right)+\mathcal{O}\left(\varepsilon^{4}\right)$. 

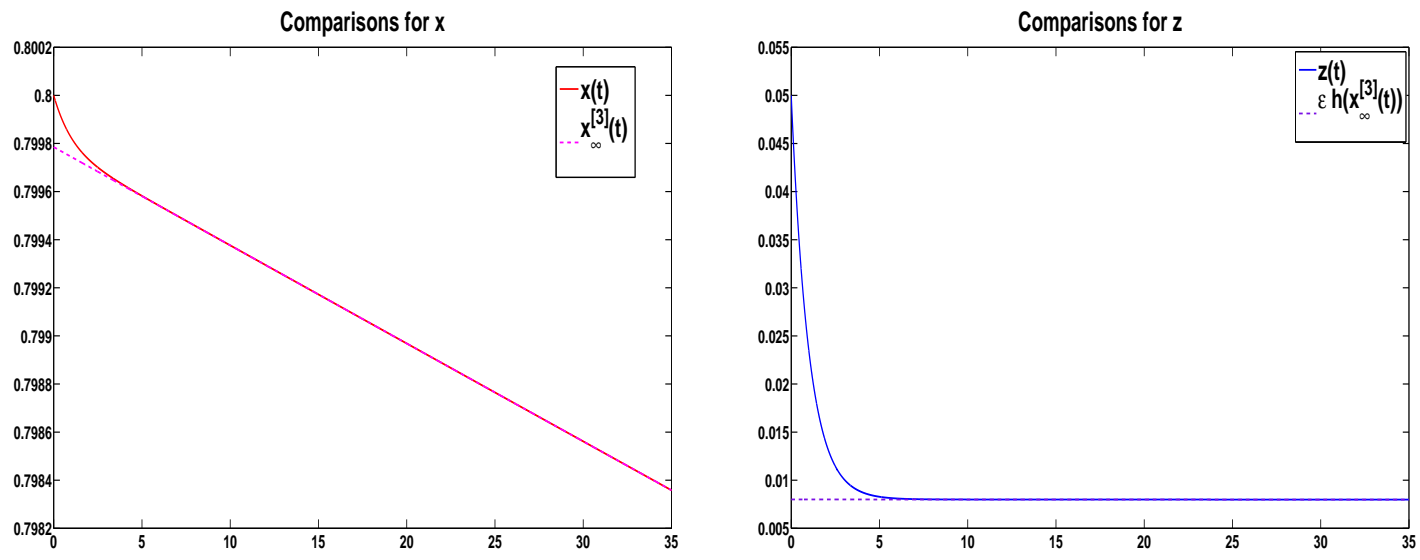

Figure 4: Comparison between $x(t)$ and $x_{\infty}^{[3]}(t)$ (left) and between $z(t)$ and $\varepsilon h\left(x_{\infty}^{[3]}(t)\right)$ (right).
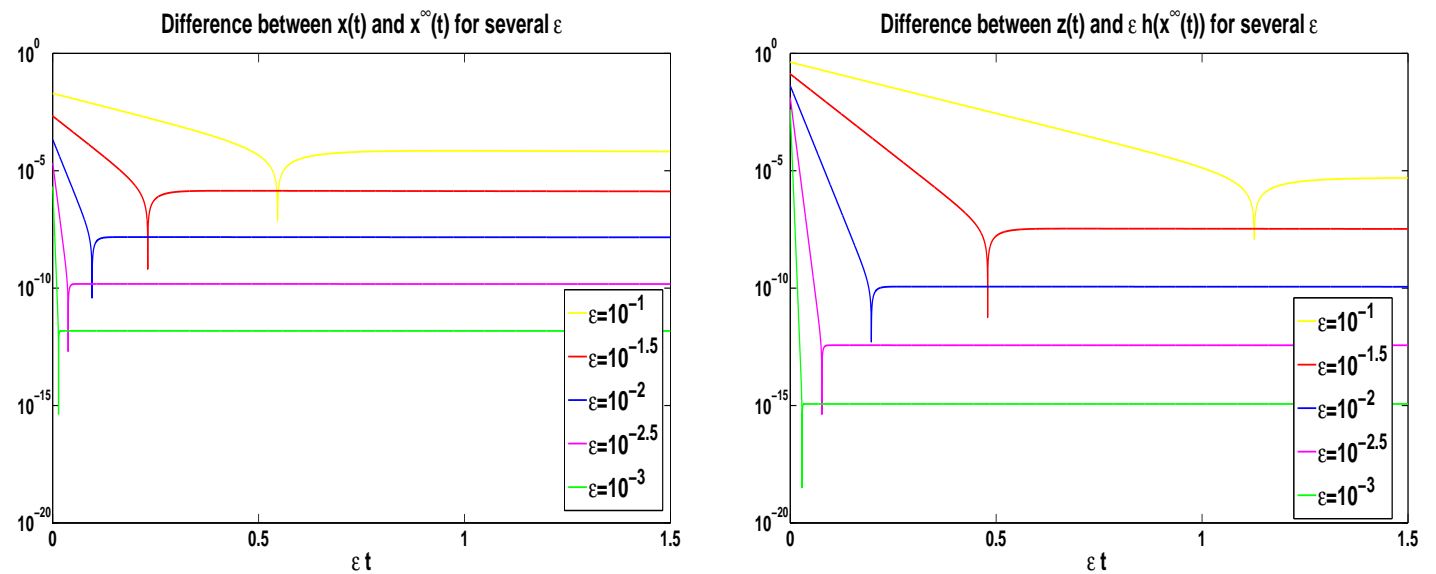

Figure 5: Comparison between $x(t)$ and $x_{\infty}^{[3]}(t)$ (left) and between $z(t)$ and $\varepsilon h\left(x_{\infty}^{[3]}(t)\right)$ (right).

Eventually, we simulate numerically the solution of the truncated normal form derived in Theorem 2.25. More precisely, Figure 6 represents the solution of the following system

$$
\left\{\begin{array}{l}
\dot{x}_{\infty}^{[3]}=\varepsilon f\left(x_{\infty}^{[3]}, \varepsilon h^{[3]}\left(x_{\infty}^{[3]}\right)\right) \\
\dot{\tilde{y}}^{[3]}=\varepsilon G^{[3]}\left(x_{\infty}^{[3]}, \tilde{y}^{[3]}\right)
\end{array}\right.
$$

with initial conditions $x^{\infty}(0)=x_{0}^{\varepsilon}, \tilde{y}(0)=z_{0}, \tilde{z}^{[3]}(t)=e^{-t} \tilde{y}^{[3]}(t)$, and where $\varepsilon G^{[3]}$ denotes the thirdorder truncation of $\varepsilon G$. Note that, in order to write down $\varepsilon G^{[2]}$, only eight trees of orders less than or equal to 2 have to be considered and their corresponding coefficients $\dot{\tilde{\delta}}(0)$ and elementary differentials computed: they are listed in Table 13. Getting the terms of order 3 is totally straightforward, though definitely more painful:

$$
\varepsilon G^{[3]}(x, y)=\varepsilon^{2}\left(x^{3} y-x^{2} y\right)+\varepsilon^{3}\left(2 x^{3}-\frac{1}{2} x^{3} y^{2}-2 x^{4}\right)=\varepsilon G(x, y)+\mathcal{O}\left(\varepsilon^{4}\right) .
$$

According to Theorem 2.25, it remains to approximate the change of variables $\Phi_{t}$ up to order 3 in $\varepsilon$, 


\begin{tabular}{|c|c|c|c|c|c|c|c|c|}
\hline$v \in \mathcal{T}_{\circ}$ & $\circ_{0}$ & $\circ_{2}$ & $\dot{\delta}_{0}^{1}$ & $\dot{\delta}_{0}^{3}$ & $\dot{\delta}_{2}^{1}$ & $\dot{\delta}_{2}^{3}$ & $\delta_{2}^{0}$ & $\delta_{2}^{2}$ \\
\hline$\tilde{\delta}_{v}(0)$ & 0 & 0 & -1 & 0 & 0 & 0 & 1 & 0 \\
\hline $\mathcal{F}_{v}(x, y)$ & $x$ & $\frac{1}{2} x y^{2}$ & $-x^{3} y$ & $\frac{1}{3} x^{3} y^{3}$ & $\frac{1}{2} x^{3} y^{3}$ & $-\frac{1}{6} x^{5} y^{5}$ & $-x^{2} y$ & $\frac{1}{2} x^{2} y^{3}$ \\
\hline
\end{tabular}

Table 13: Coefficients $\dot{\tilde{\delta}}(0)$ and elementary differentials for trees of $\mathcal{T}_{\circ}$ with order $\leq 2$.

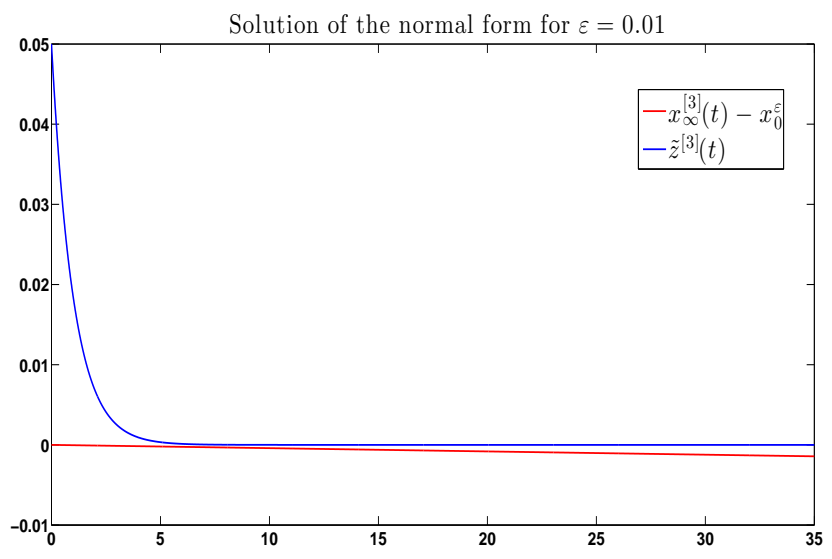

Figure 6: Solution $\left(x_{\infty}^{[3]}(t), \tilde{z}^{[3]}(t)\right)$ of (3.3) for $x_{0}=0.8, z_{0}=0.05$ and $\varepsilon=0.01$.

a task that necessitates the values of coefficients $\delta(0, t)$, presented in Table 14 for trees of orders less than or equal to 2 . We shall omit the other details of the calculations and define

$$
\Phi_{t}^{[3]}=\Phi_{t}+\mathcal{O}\left(\varepsilon^{4}\right)
$$

Denoting

$$
\left(x^{[3]}(t), y^{[3]}(t)\right)=\Phi_{t}^{[3]} \circ\left(x_{\infty}^{[3]}(t), \tilde{y}^{[3]}(t)\right)=(x(t), y(t))+\mathcal{O}\left(\varepsilon^{4}\right),
$$

and $z^{[3]}(t)=e^{-t} y^{[3]}(t)$, we are finally in a position to represent the differences $x(t)-x^{[3]}(t)$ (left) and $z(t)-z^{[3]}(t)$ (right) on Figure 7 for various values of $\varepsilon$. A fourth-order (in $\varepsilon$ ) error is clearly observed for all time in accordance with what Theorem 2.25 predicts.

\subsection{A slow manifold with oscillatory dynamics}

The second system we consider has higher dimension (3) and is written

$$
\left\{\begin{array}{l}
\dot{x}=\varepsilon(1-z)\left(\begin{array}{cc}
0 & -1 \\
1 & 0
\end{array}\right) x \\
\dot{z}=-z+\varepsilon x_{1}^{2} x_{2}^{2}
\end{array}\right.
$$

with $x_{0}=\left(\begin{array}{l}0.1 \\ 0.7\end{array}\right)$ and $z_{0}=0.05$. Figure 8 (left) represents the different components of its exact solution for $\varepsilon=0.01$. The complete trajectory and its projection onto the $\left(x_{1}, x_{2}\right)$-plane are represented on Figure 9. As for previous example, all functions $f_{k}$ and $g_{k}$ vanish at the exception of 


\begin{tabular}{|c|c|c|c|c|c|c|}
\hline$u \in \mathcal{T}_{\bullet}$ & $\bullet_{1}$ & $\bullet$ & $\dot{\sigma}_{1}^{3}$ & $\dot{d}_{0}^{1}$ & $\dot{\phi}_{1}^{1}$ & $\dot{0}^{3}$ \\
\hline$\delta_{u}(0, t)$ & $-e^{-t}$ & $-1 / 3 e^{-3 t}$ & $1 / 12 e^{-4 t}$ & $1 / 4 e^{-4 t}$ & $1 / 2 e^{-2 t}$ & $1 / 12 e^{-6 t}$ \\
\hline $\mathcal{F}_{u}(x, y)$ & $-x^{3} y$ & $\frac{1}{3} x^{3} y^{3}$ & $-x^{5} y^{4}$ & $-x^{5} y^{4}$ & $3 x^{5} y^{2}$ & $\frac{1}{3} x^{5} y^{6}$ \\
\hline$w \in \mathcal{T}$ & $\rho_{\cdot}^{0}$ & $\rho_{0}^{2}$ & 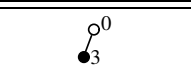 & $\int_{0}^{2}$ & $\rho_{2}^{0}$ & $\rho_{2}^{2}$ \\
\hline$\delta_{w}(0, t)$ & $e^{-t}$ & $\frac{1}{2} e^{-2 t}-e^{-t}$ & $\frac{1}{3} e^{-3 t}-\frac{1}{2} e^{-2 t}$ & $\frac{1}{4} e^{-4 t}-\frac{1}{3} e^{-3 t}$ & $e^{-t}-1$ & $\frac{1}{2}+\frac{1}{2} e^{-2 t}-e^{-t}$ \\
\hline $\mathcal{F}_{w}(x, y)$ & $x^{4}$ & $\frac{1}{2} x^{4} y^{2}$ & $x^{4} y^{2}$ & $-\frac{1}{2} x^{4} y^{4}$ & $-x^{2} y$ & $\frac{1}{2} x^{2} y^{3}$ \\
\hline$\overline{v v \in \mathcal{T}_{\circ}}$ & $\circ_{0}$ & $\mathrm{o}_{2}$ & $\int_{0}^{1}$ & $\dot{\delta}_{0}^{3}$ & $d_{2}^{1}$ & $\delta_{2}^{3}$ \\
\hline$\delta_{v}(0, t)$ & $-1+e^{t}$ & $1-e^{-t}$ & 0 & $-\frac{1}{6}\left(1-e^{-2 t}\right)$ & $-\frac{1}{2}\left(1-e^{-2 t}\right)$ & $-\frac{1}{12}\left(1-e^{-4 t}\right)$ \\
\hline $\mathcal{F}_{v}(x, y)$ & $x$ & $\frac{1}{2} x y^{2}$ & $-x^{3} y$ & $\frac{1}{3} x^{3} y^{3}$ & $\frac{1}{2} x^{3} y^{3}$ & $-\frac{1}{6} x^{5} y^{5}$ \\
\hline
\end{tabular}

Table 14: Coefficients $\delta(0, t)$ and elementary differentials for trees of $\mathcal{T}$ with order $\leq 2$.
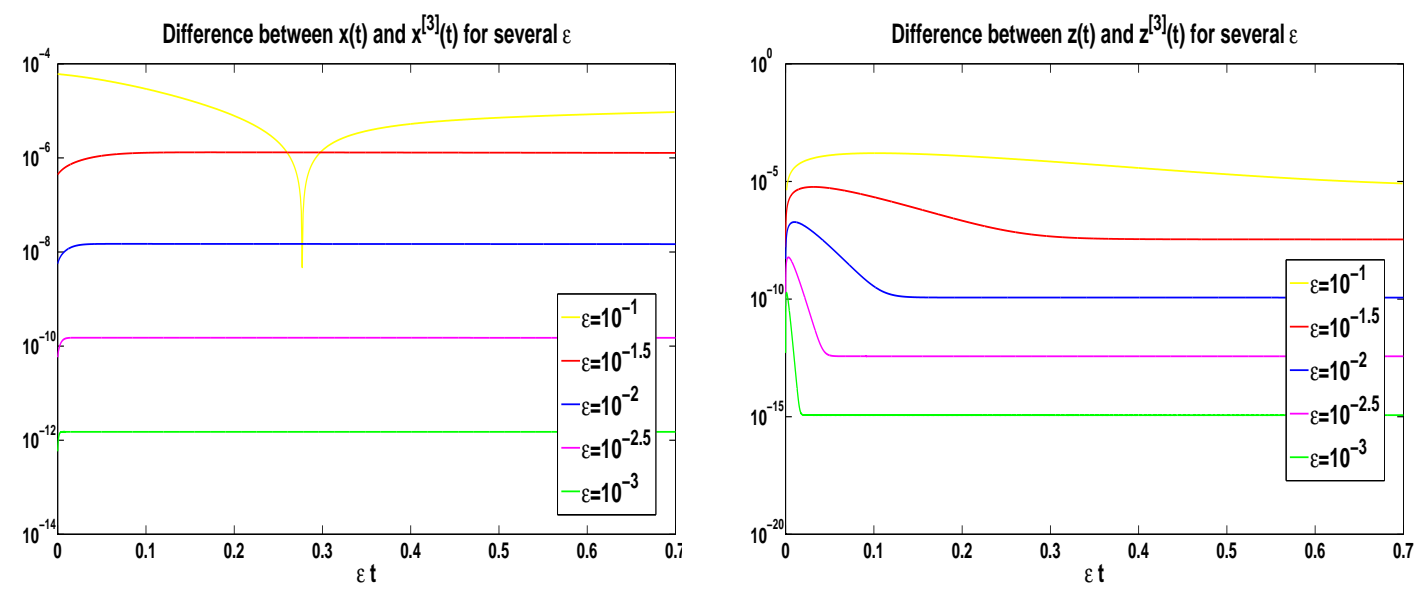

Figure 7: Comparison between $x(t)$ and $x^{[3]}(t)$ (left) and between $z(t)$ and $z^{[3]}(t)$ (right).

$$
\begin{aligned}
& f_{0}(x, z)=f(x, 0)=\left(\begin{array}{cc}
0 & -1 \\
1 & 0
\end{array}\right) x, \quad f_{1}(x, z)=\partial_{z} f(x, 0) z=-z\left(\begin{array}{cc}
0 & -1 \\
1 & 0
\end{array}\right) x \\
& g_{0}(x, z)=g(x, 0)=x_{1}^{2} x_{2}^{2} .
\end{aligned}
$$

Proceeding as for previous example, we obtain the elements of Table 15, where we have denoted $J=\left(\begin{array}{cc}0 & -1 \\ 1 & 0\end{array}\right)$, and upon computing third-order terms in a similar way we arrive at the following truncated expansion of $x_{0}^{\varepsilon}$

$$
\begin{aligned}
x_{0}^{\varepsilon}=( & \left.-\frac{\varepsilon^{2}}{2} y_{0}^{2}+\frac{\varepsilon^{3}}{2} x_{0,1}^{2} x_{0,2}^{2} y_{0}\right) x_{0}+\left[-\varepsilon y_{0}+\varepsilon^{2} x_{0,1}^{2} x_{0,2}^{2}\right. \\
& \left.+\varepsilon^{3}\left(\frac{1}{6} y_{0}^{3}+2\left(1+2 y_{0}\right)\left(x_{0,1} x_{0,2}^{3}-x_{0,1}^{3} x_{0,2}\right)\right)\right] J x_{0}+\mathcal{O}\left(\varepsilon^{4}\right) .
\end{aligned}
$$



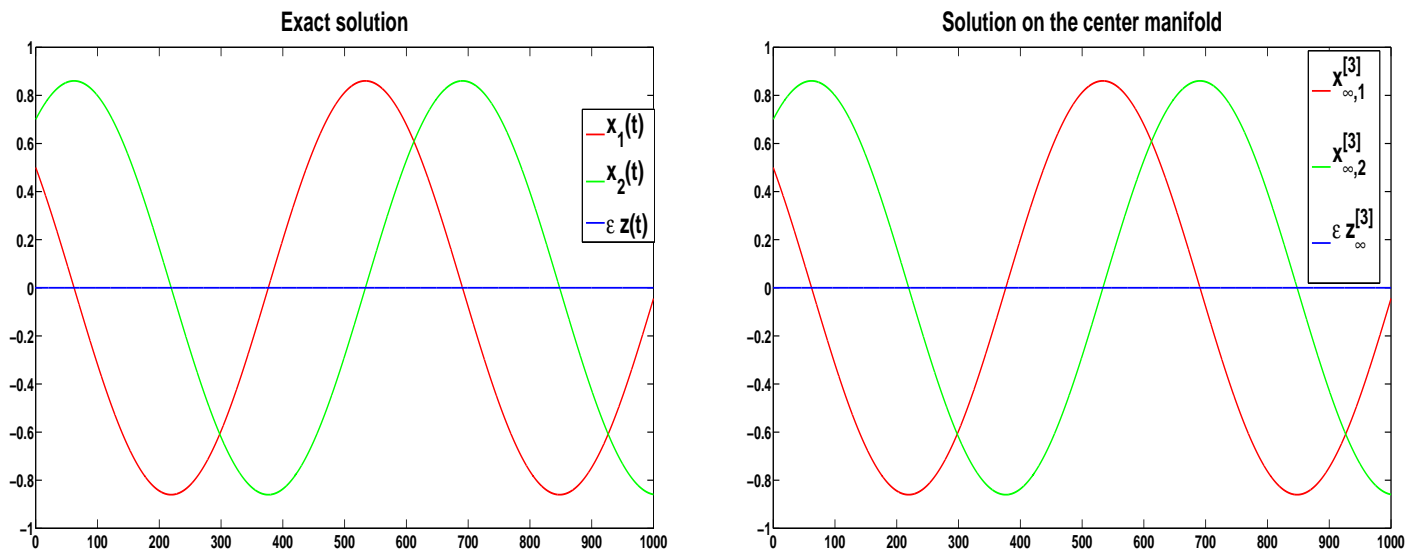

Figure 8: Exact solution for $\varepsilon=0.01, x_{0}=(0.5 ; 0.7)^{T}$ and $z_{0}=0.05$ (left) and solution $\left(x_{\infty}^{[3]}(t), z_{\infty}^{[3]}(t)\right)$ of (3.5) (right).
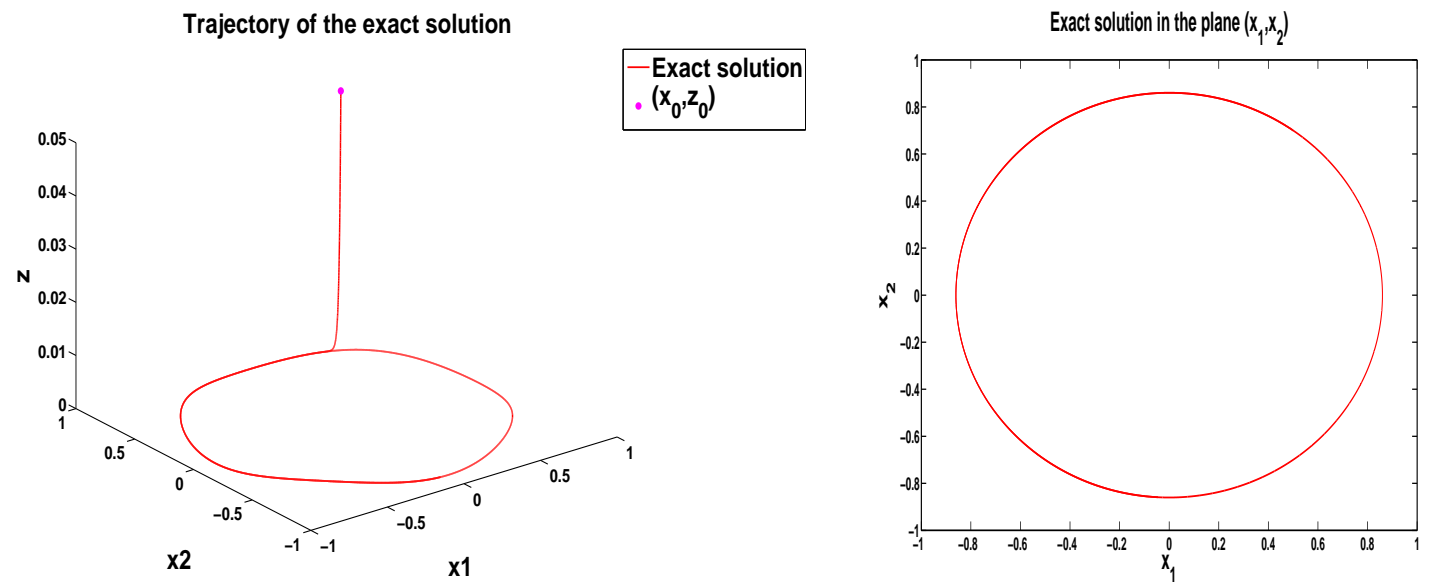

Figure 9: Exact trajectory and its projection onto the $\left(x_{1}, x_{2}\right)$-plane.

The center manifold is simply here

$$
\begin{aligned}
\varepsilon h^{[3]}(x) & :=\varepsilon x_{1}^{2} x_{2}^{2}-2 \varepsilon^{2}\left(x_{1}^{3} x_{2}-x_{2}^{3} x_{1}\right)+\varepsilon^{3}\left(-4 x_{1} x_{2}+2\left(x_{1}^{3} x_{2}-x_{2}^{3} x_{1}\right)+2\left(x_{1}^{4}-4 x_{1}^{2} x_{2}^{2}+x_{2}^{4}\right)\right) \\
& =\varepsilon h(x)+\mathcal{O}\left(\varepsilon^{4}\right) .
\end{aligned}
$$

On the right of Figure 8 is represented the exact solution of the system

$$
\left\{\begin{aligned}
\dot{x}_{\infty}^{[3]} & =\varepsilon f\left(x_{\infty}^{[3]}, \varepsilon h^{[3]}\left(x_{\infty}^{[3]}\right)\right) \\
z_{\infty}^{[3]} & =\varepsilon h^{[3]}\left(x_{\infty}^{[3]}\right)
\end{aligned}\right.
$$

with initial condition $x_{\infty}^{[3]}(0)=x_{0}^{\varepsilon}$. Finally, Figure 10 represents the quantities $x_{1}(t)-x_{\infty, 1}^{[3]}(t)$ and $x_{2}(t)-x_{\infty, 2}^{[3]}(t)$, while Figure 11 draws the difference $z(t)-\varepsilon h^{[3]}\left(x_{\infty}^{[3]}(t)\right)$, for several values of $\varepsilon$. They demonstrate a clear-cut numerical confirmation of Theorem 2.21. 


\begin{tabular}{|c|c|c|c|c|c|c|c|}
\hline$u \in \mathcal{T}_{\bullet}$ & $\bullet_{0}$ & $\bullet_{1}$ & $\dot{\bullet}_{0}^{0}$ & $\dot{\bullet}_{0}^{1}$ & $\dot{\bullet}_{1}^{0}$ & $\dot{\bullet}_{1}^{1}$ & $\stackrel{0}{0}_{1}^{0}$ \\
\hline$\tilde{\gamma}_{u}(0)$ & 0 & 1 & 0 & -1 & 1 & $1 / 2$ & -1 \\
\hline $\mathcal{F}_{u}(x, y)$ & $J x$ & $-y J x$ & $-x$ & $y x$ & $y x$ & $-y^{2} x$ & $-x_{1}^{2} x_{2}^{2} J x$ \\
\hline
\end{tabular}

Table 15: Coefficients $\tilde{\gamma}(0)$ and elementary differentials for trees of $\mathcal{T}_{\bullet}$ with order $\leq 2$.
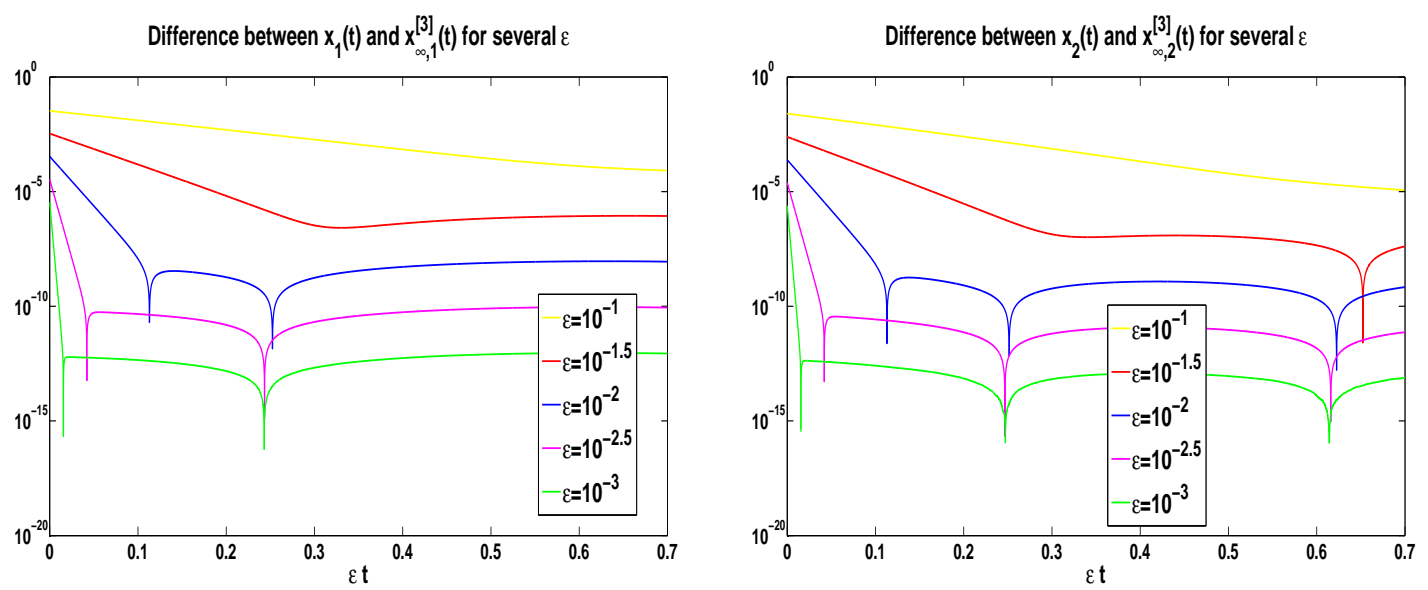

Figure 10: Error between $x_{1}(t)$ and $x_{\infty, 1}^{[3]}(t)$, and between $x_{2}(t)$ and $x_{\infty, 2}^{[3]}(t)$.

Computing the numerical solution of the truncated normal form of Theorem 2.25 leads to Figure 11 , which represents the components of the following system

$$
\left\{\begin{array}{l}
\dot{x}_{\infty}^{[3]}=\varepsilon f\left(x_{\infty}^{[3]}, \varepsilon h^{[3]}\left(x_{\infty}^{[3]}\right)\right) \\
\dot{\tilde{y}}^{[3]}=\varepsilon G^{[3]}\left(x_{\infty}^{[3]}, \tilde{y}^{[3]}\right)
\end{array}\right.
$$

with initial conditions $x^{\infty}(0)=x_{0}^{\varepsilon}, \tilde{y}(0)=z_{0}$ and $\tilde{z}^{[3]}(t)=e^{-t} \tilde{y}^{[3]}$. The coefficients $\dot{\tilde{\delta}}(0)$ associated with $\varepsilon G^{[2]}$ are listed in Table 16 and further computations lead to

\begin{tabular}{|c|c|c|c|}
\hline$v \in \mathcal{T}_{\circ}$ & $0_{0}$ & $\dot{\delta}_{0}^{0}$ & $\dot{\delta}_{0}^{1}$ \\
\hline$\tilde{\tilde{\delta}}_{v}(0)$ & 0 & 1 & -1 \\
\hline $\mathcal{F}_{v}(x, y)$ & $x_{1}^{2} x_{2}^{2}$ & $-2\left(x_{1} x_{2}^{3}-x_{1}^{3} x_{2}\right)$ & $2\left(x_{1} x_{2}^{3}-x_{1}^{3} x_{2}\right) y$ \\
\hline
\end{tabular}

Table 16: Coefficients $\dot{\tilde{\delta}}(0)$ and elementary differentials for trees of $\mathcal{T}$ 。 with order $\leq 2$.

$$
\varepsilon G^{[3]}(x, y)=-2 \varepsilon^{2}\left(x_{1} x_{2}^{3}-x_{1}^{3} x_{2}\right)(1+y)+2 \varepsilon^{3}\left(x_{1}^{2} x_{2}^{2}\left(2+x_{1} x_{2}^{3}-x_{1}^{3} x_{2}\right)-x_{1}^{4}+4 x_{1}^{2} x_{2}^{2}-x_{2}^{4}\right) .
$$

Finally, coefficients $\delta(0, t)$ and elementary differentials required for the expansion of $\Phi_{t}$ are presented in Table 17 for trees of orders less than or equal to 2. The differences $x_{1}(t)-x_{1}^{[3]}(t)$ (left) and $x_{2}(t)-x_{2}^{[3]}(t)$ (right) are drawn on Figure 12, while the difference $z(t)-z^{[3]}(t)$ is plot on Figure 13, for several values of $\varepsilon$. Again, numerical experiments clearly support the assertion of Theorem 2.25. 

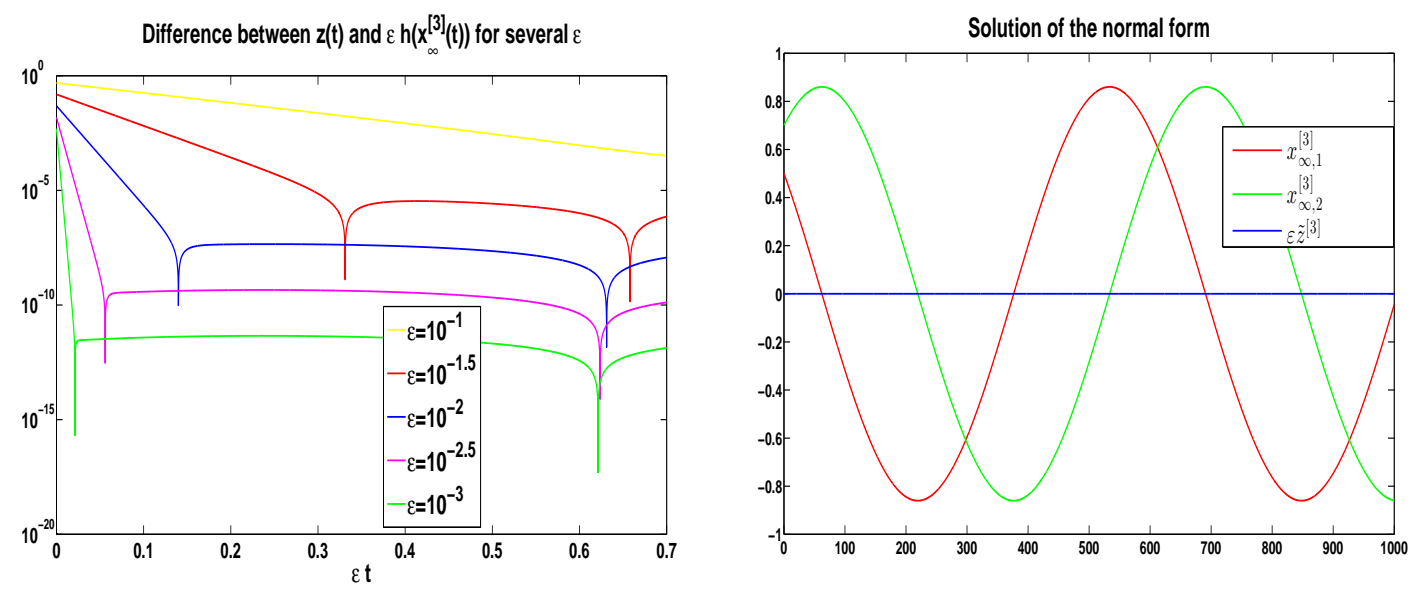

Figure 11: Error between $z(t)$ and $\varepsilon h\left(x_{\infty}^{[3]}(t)\right)$ (left) and solution $\left(x_{\infty}^{[3]}(t), \tilde{z}(t)\right)$ of $(3.6)$ for $\varepsilon=0.01$, $x_{0}=(0.5 ; 0.7)^{T}$ and $z_{0}=0.05$ (right).

\begin{tabular}{|c|c|c|c|c|c|}
\hline$u \in \mathcal{T}_{\bullet}$ & $\bullet_{0}$ & $\bullet_{1}$ & $\mathscr{d}_{0}^{0}$ & $\int_{0}^{1}$ & $\mathscr{b}_{1}^{0}$ \\
\hline$\delta_{u}(0, t)$ & 0 & $-e^{-t}$ & 0 & $\overline{e^{-t}}$ & $-e^{-t}$ \\
\hline $\mathcal{F}_{u}(x, y)$ & $J x$ & $-y J x$ & $-x$ & $y x$ & $y x$ \\
\hline$\overline{p w \in \mathcal{T}_{\circ}}$ & $\overbrace{1}^{1}$ & $\bar{c}_{\rho_{1}^{0}}$ & ${ }^{\circ} 0$ & 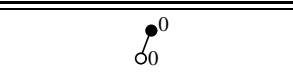 & $\overline{\delta_{0}^{1}}$ \\
\hline$\delta_{w}(0, t)$ & $\frac{1}{2} e^{-2 t}$ & $e^{-t}$ & $-1+e^{t}$ & $1-e^{t}$ & 0 \\
\hline $\mathcal{F}_{w}(x, y)$ & $-y^{2} x$ & $-x_{1}^{2} x_{2}^{2} J x$ & $x_{1}^{2} x_{2}^{2}$ & $-2\left(x_{1} x_{2}^{3}-x_{1}^{3} x_{2}\right)$ & $2\left(x_{1} x_{2}^{3}-x_{1}^{3} x_{2}\right) y$ \\
\hline
\end{tabular}

Table 17: Coefficients $\delta(0, t)$ and elementary differentials for trees of $\mathcal{T}$ with order $\leq 2$.

\section{Future work}

In the process of reduction of (1.1) to its center-manifold form, it is of paramount importance to determine the correct new initial condition $x_{0}^{\varepsilon}$. Furthermore, if some information is to be extracted from this reduced system in the transient phase, it is necessary to add a complementary equation and then to apply an appropriate change of variables. All the transformations introduced to this aim in this paper have been developed in terms of B-series, the convergence of which is usually not ensured. It is thus our intention to analyze truncation errors and their impact in a future paper. Of much interest also, in our opinion, is the extension of these results to the situation where problem (1.1) involves different eigenvalues $\lambda_{i}$. Finally, we think that a better understanding of the structure of the center-manifold equations will lead to new numerical schemes ${ }^{7}$ and this is the reason why B-series, which can represent virtually all numerical methods, have been preferred here to word-series.

\footnotetext{
${ }^{7}$ In the same way that [CMSS10] has paved the way for [CCMSS11, CMMV15].
} 

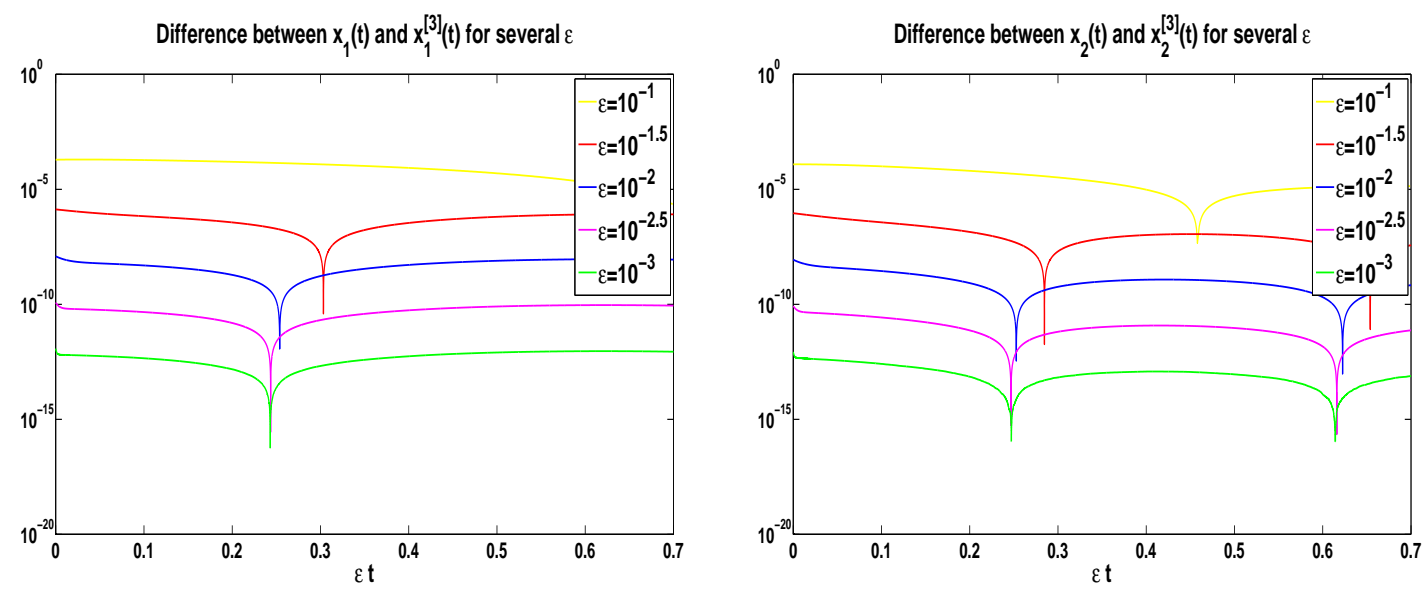

Figure 12: Comparison between $x_{1}(t)$ and $x_{1}^{[3]}(t)$ (left) and between $x_{2}(t)$ and $x_{2}^{[3]}(t)$ (right).

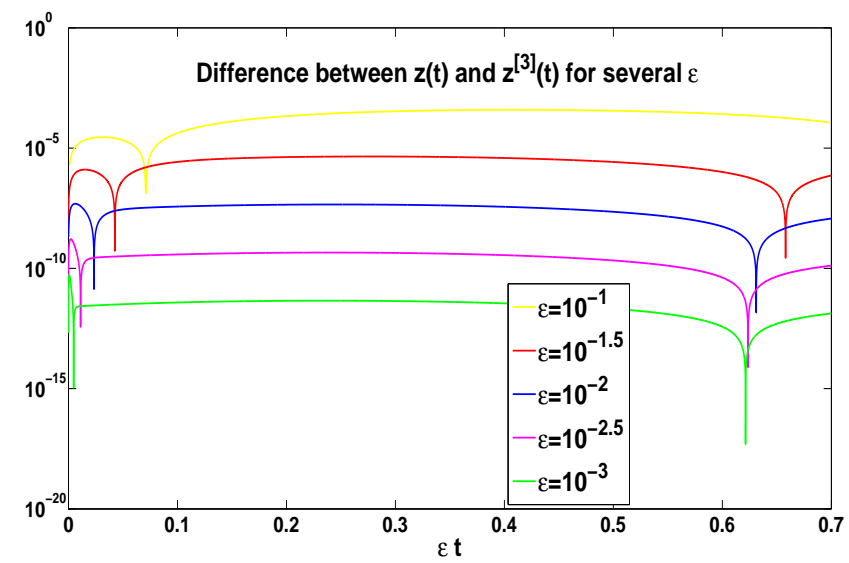

Figure 13: Comparison between $z(t)$ and $z^{[3]}(t)$.

\section{References}

[AR94] P. Auger and R. Roussaries. Complex ecological models with simple dynamics : from individual to populations. Acta Biotheoretica, 42:111-136, 1994.

[But72] J.C. Butcher. An algebraic theory of integration methods. Computational mathematics, 26:79-106, 1972.

[But87] J.C. Butcher. The numerical analysis of ordinary differential equations. WileyInterscience Publication, 1987.

[Car81] J. Carr. Applications of Centre Manifold Theory, volume 35 of Applied Mathematical Sciences Series. Springer-Verlag, 1981.

[CCMSS11] M.P. Calvo, P. Chartier, A. Murua, and J.M. Sanz-Serna. Numerical experiments with the stroboscopic method. Appl. Numer. Math., 61:1077-1095, 2011. 
[CCS] F. Castella, P. Chartier, and J. Sauzeau. Analysis of a time-dependent problem of mixed migration and population dynamics.

[CHL09] F. Castella, J.P Hoffbeck, and Y. Lagadeuc. A reduced model for spatially structured predator-prey systems with fast spatial migrations and slow demographic evolutions. Asymptotic Analysis, 61(3-4):125-175, 2009.

[CHV10] P. Chartier, E. Hairer, and G. Vilmart. Algebraic structures of B-series. Foundations of Computational Mathematics, 10:407-427, 2010.

[CMMV15] P. Chartier, J. Makazaga, M. Murua, and G. Vilmart. Multi-revolution composition methods for highly oscillatory differential equations. Numerische Mathematik, 128(1):167_ 192, 2015.

[CMSS10] P. Chartier, A. Murua, and J.M. Sanz-Serna. Higher order averaging, formal series and numerical integration I: B-series. Foundations of Computational Mathematics, 10:695$727,2010$.

[CMSS12a] P. Chartier, A. Murua, and J.M. Sanz-Serna. A formal series approach to averaging: exponentially small error estimates. Discrete ans Continuous Dynamical Systems, 32:3009-3027, 2012.

[CMSS12b] P. Chartier, A. Murua, and J.M. Sanz-Serna. Higher order averaging, formal series and numerical integration II: the quasi-periodic case. Foundations of Computational Mathematics, 12:471-508, 2012.

[CMSS15] P. Chartier, A. Murua, and J.M. Sanz-Serna. Higher order averaging, formal series and numerical integration III: error bounds. Foundations of Computational Mathematics, 15:591-612, 2015.

[HLW06] E. Hairer, C. Lubich, and G. Wanner. Geometric numerical integration. Structure preserving algorithms for ordinary differential equations, volume 31 of Computational Mathematics. Springer-Verlag, 2006.

[MSS16a] A. Murua and J.M. Sanz-Serna. Averaging and computing normal forms with word series algorithms. 2016.

[MSS16b] A. Murua and J.M. Sanz-Serna. Computing normal forms and formal invariants of dynamical systems by means of word series. Nonlinear Analysis, Theory, Methods and Applications, 2016.

[MSS16c] A. Murua and J.M. Sanz-Serna. Word series for dynamical systems and their numerical integrators. Foundations of Computational Mathematics, 2016.

[PA96] J.C. Poggiale and P. Auger. Fast oscillating migations in a predator-prey model. Mathematical models and methods in applied sciences, 6(2):271-226, 1996. 